Submitted to ApJ

Preprint typeset using LTEX style emulateapj v. 01/23/15

\title{
COLD FILAMENTARY ACCRETION AND THE FORMATION OF METAL POOR GLOBULAR CLUSTERS AND HALO STARS
}

\author{
Nir Mandelker ${ }^{1,2,3}$, Pieter G. VAn DokKum ${ }^{2}$, JeAn P. Brodie ${ }^{4}$, Frank C. VAn den Bosch ${ }^{2}$, Daniel Ceverino 5 \\ Submitted to ApJ
}

\begin{abstract}
We propose that cold filamentary accretion in massive galaxies at high redshift can lead to the formation of star-forming clumps in the halos of these galaxies without the presence of dark matter sub-structure. In certain cases, these clumps can be the birth places of metal poor globular-clusters (MP GCs). Using cosmological simulations, we show that narrow streams of dense gas feeding massive galaxies from the cosmic web can fragment, producing star-forming clumps. We then derive an analytical model for the properties of streams as a function of halo mass and redshift, and assess when these are gravitationally unstable, when this can lead to collapse and star-formation in the halo, and when it may result in the formation of MP GCs. For stream metalicities $\gtrsim 0.01 Z_{\odot}$, this is likely to occur at $z>4.5$. At $z \sim 6$, the collapsing clouds have masses of $\sim 5-10 \times 10^{7} M_{\odot}$ and the average stream pressure is $\sim 10^{6} \mathrm{~cm}^{-3} \mathrm{~K}$. The conditions for GC formation are met in the extremely turbulent "eyewall" at $\sim 0.3 R_{\mathrm{v}}$, where counter-rotating streams can collide, driving very large densities. Our scenario can account for the observed kinematics and spatial distribution of MP GCs, the correlation between their mass and metalicity, and the mass ratio between the GC system and the host halo. For MW mass halos, we infer that $\sim 30 \%$ of MP GCs could have formed in this way, the remainder likely accreted in mergers. Our predictions for GC formation along circumgalactic filaments at high-redshift are testable with JWST.
\end{abstract}

Keywords: globular clusters: general — galaxies: formation — instabilities

\section{INTRODUCTION}

The origin of globular clusters (GCs) has long challenged models of galaxy formation. GCs are bi-modal, with blue, metal-poor (MP), and red, metal-rich (MR), sub-populations (Larsen et al. 2001; Brodie \& Strader 2006; Brodie et al. 2012). The distribution of metalicities for the two populations typically peak at $[\mathrm{Fe} / \mathrm{H}] \sim-1.5$ and -0.5 respectively, though both peaks tend to shift to higher $[\mathrm{Fe} / \mathrm{H}]$ with increasing galaxy luminosity (Brodie \& Huchra 1991; Brodie \& Strader 2006; Peng et al. 2006; Forte et al. 2009; Kruijssen 2014). In the Milky-Way, the populations are typically divided at $[\mathrm{Fe} / \mathrm{H}] \sim-1.1$. MP GCs typically comprise $\sim 0.5-0.67$ of the total GC population, with the fraction even higher in low mass dwarfs (Strader et al. 2006; Peng et al. 2006). Both populations have comparable ages, to within measurement errors, roughly $12.5 \mathrm{Gyr} \pm$ 1 Gyr (e.g. Marín-Franch et al. 2009; VandenBerg et al. 2013; Forbes et al. 2015), though most models suggest that MP GCs formed on average earlier than MR ones (e.g. Forbes et al. 2015). This corresponds to GCs having formed before $z \sim 3$, possibly even before the end of reionization.

The mass functions of both MP and MR GCs are roughly the same. Both have a log-normal distribution, with typical masses in the range $\sim 10^{4}-10^{6} \mathrm{M} \odot$ and an average mass of $\sim 2 \times 10^{5}$ (Brodie \& Strader 2006; Wehner et al. 2008;

\footnotetext{
${ }^{1}$ corresponding author: nir.mandelker@yale.edu

${ }^{2}$ Department of Astronomy, Yale University, PO Box 208101, New Haven, CT, USA

${ }^{3}$ Heidelberger Institut für Theoretische Studien, SchlossWolfsbrunnenweg 35, 69118 Heidelberg, Germany

${ }^{4}$ University of California Observatories, 1156 High Street, Santa Cruz, CA 95064, USA

${ }^{5}$ Universität Heidelberg, Zentrum für Astronomie, Institut für Theoretische Astrophysik, Albert-Ueberle-Str. 2, 69120 Heidelberg, Germany
}

Harris et al. 2013). The cutoff at the low mass end is thought to be due to disruption and evaporation of low mass GCs since their formation, while the initial mass function may have been a power law (Elmegreen \& Efremov 1997; Elmegreen 2010; Baumgardt 1998; Fall \& Zhang 2001; Kravtsov \& Gnedin 2005; Prieto \& Gnedin 2008; McLaughlin \& Fall 2008; Kruijssen 2015). However, others have argued that the tidal forces experienced by GCs in the early Universe may have been much weaker than assumed, and thus that it is unlikely that a power law initial mass function could have been transformed into a log-normal distribution (Gieles \& Renaud 2016; Renaud et al. 2017). Regardless, the surviving GCs should have undergone mass loss since their formation. Models attempting to explain chemical abundance anomalies in GCs by invoking multiple stellar populations require the mass at formation to be $>20$ times more massive than present day masses (see Kruijssen 2014 for a recent review of such models). However, empirical models based on the observational consequences of such massive proto GCs suggest the mass loss ratio must be less than 10 (Boylan-Kolchin 2017), while analytical models of the physics of GC disruption predict mass loss ratios as low as $\sim 3$ (Fall \& Zhang 2001; Kruijssen 2015), which is also found in N-body simulations of cluster disruption (Webb \& Leigh 2015). MP and MR GCs have comparable sizes, with half light radii of $\sim 2-3 \mathrm{pc}$ (Brodie \& Strader 2006).

The MP GCs are particularly enigmatic objects, with some very puzzling properties. Their spatial distribution often appears even more extended than the stellar halo (Strader et al. 2011; Forbes et al. 2012; Durrell et al. 2014; Kruijssen 2014), suggesting that most of them did not form inside their host galaxies. The fraction of MP GCs outside of $\sim 10 \mathrm{kpc}$ is much higher than in the central $\sim 5 \mathrm{kpc}$ of galaxies (Harris et al. 2006). By contrast, MR GCs fol- 
low the galaxy light and are associated with the stellar disk and bulge, suggesting that they formed along with the thick disk and the bulge, perhaps during an unstable clumpy phase (Elmegreen et al. 2008; Shapiro et al. 2010; Kruijssen 2015; Renaud et al. 2017; Mandelker et al. 2017), or following wet mergers at intermediate redshifts (Ashman \& Zepf 1992; Kravtsov \& Gnedin 2005; Li \& Gnedin 2014; Li et al. 2017). MP GCs exhibit a mass-metalicity relation, known as the "blue tilt", such that more massive MP GCs are more metal rich (Strader et al. 2006; Peng et al. 2006; Spitler et al. 2006; Harris et al. 2006). This may be due to self-enrichment of massive MP GCs (Harris et al. 2006; Strader \& Smith 2008; Bailin \& Harris 2009), but the mechanism may depend on environment and does not appear universal (Spitler et al. 2006; Strader et al. 2006; Brodie \& Strader 2006). No such relation is observed for MR GCs (Brodie \& Strader 2006; Wehner et al. 2008). The kinematics of the two populations are also different, with the population of MP GCs showing more tangential orbits with significant apparent rotation, as opposed to the mostly radial orbits expected if they were mainly accreted (Pota et al. 2013, 2015a.b). The tangential anisotropy of MP GCs increases with distance from the halo center (Agnello et al. 2014). The MR GCs, on the other hand, have more mixed orbits (Pota et al. 2015b).

One of the most puzzling properties of GCs is that the total mass of the GC system (GCS) in a galaxy is a near constant fraction of the dark matter halo mass, with a ratio of $\sim 2.9 \times 10^{-5} \pm 0.28$ dex (Hudson et al. 2014; Harris et al. 2015, 2017). This is in stark contrast to the highly nonlinear relation between a galaxy's stellar mass and halo mass (e.g. Yang et al. 2003; Behroozi et al. 2013). While a linear relation between GCS mass and halo mass exists for the total GC population, it appears mainly driven by the MP GCS (Harris et al. 2015). This relation holds over 5 orders of magnitude in galaxy mass, and in extreme environments, such as entire clusters of galaxies and Ultra-Diffuse Galaxies (UDGs) (Harris et al. 2017; van Dokkum et al. 2017). Some have suggested that this relation is a coincidence, resulting from a stellar mass dependent destruction efficiency for GCs combined with the non-linear stellar-to-halo mass relation (Kruijssen 2015, though see Fall \& Chandar 2012 for evidence against a mass dependent destruction efficiency for clusters in the local Universe) or as a result of hierarchical galaxy assembly and the central limit theorem (El-Badry et al. 2018). However, many others have pointed out that this is suggestive of a link between GC formation and halo assembly at high redshift (e.g Spitler \& Forbes 2009; Harris et al. 2017; Boylan-Kolchin 2017).

Recent measurements indicate that the radial extent of GCSs, as measured by their half-number radii, is a constant fraction of the halo virial radius, $R_{\mathrm{GC}} \sim 0.04 R_{\mathrm{v}}$ (Forbes 2017). This may be further evidence for an intimate connection betweent the properties of GCSs and their dark matter host halos. However, Hudson \& Robison (2017) found a non-linear relation between the sizes of GCSs and the virial radii of their host halos, albeit with a smaller sample than Forbes (2017). Further observations are needed to clarify this point.

Several classes of models have been envoked to account for the formation of MP GCs. Some models propose that they form at the centres of dark matter halos at the earliest stages of galaxy formation, prior to reionization (Peebles 1984; Rosenblatt et al. 1988; Moore et al. 2006). However, there is no dynamical evidence for dark matter in GCs (Moore
1996; Conroy et al. 2011). A second class of models predict that GCs formed within the gaseous halos surrounding massive galaxies in the early Universe, as opposed to in the halo centres, due to instabilities in the halo (e.g. Fall \& Rees 1985; Cen 2001; Scannapieco et al. 2004). A third class of models suggests that MP GCs formed in dwarf galaxies in the early Universe, possibly as a result of major mergers (Kravtsov \& Gnedin 2005; Li \& Gnedin 2014; Li et al. 2017; Kim et al. 2017). These then merged onto larger galaxies and deposited their GCs in the halos of their new hosts (Ashman \& Zepf 1992; Kravtsov \& Gnedin 2005; Muratov \& Gnedin 2010; Elmegreen et al.|2012; Kimm et al. 2016; Kim et al. 2017; Renaud et al. 2017). The similarity between properties of GCs and those of young massive clusters (YMCs) in the local Universe has led to the suggestion that GCs may be the descendants of ordinary YMC formation at high redshift (Elmegreen \& Efremov 1997; Kravtsov \& Gnedin 2005; Prieto \& Gnedin 2008; Kruijssen 2014, 2015, though see also Renaud et al. 2017). Kruijssen (2015) argues that GC formation is a two stage process, beginning with a rapid-disruption phase in the high-pressure environments of high redshift discs until mergers cause them to migrate out into the halo, followed by slow evaporation in the halos. While this model is able to reproduce many observed properties of GCs and GCSs assuming that all GCs formed at $z \sim 3$, it is not at all clear that all GCs formed inside galaxy discs, and other formation mechanisms should be explored (see discussion in Kruijssen 2014).

In particular, the observed connection between GCSs and their dark-matter host halos warrents further investigation as to whether such a relation could have existed at their formation. Recently, an empirical model has been proposed where MP GCs form directly in their dark matter host halos at $z \gtrsim 6$ in direct proportion to the host halo mass, and then undergo subsequent hierarchical merging of halos and of GCSs (Boylan-Kolchin 2017). It was shown that this can yield $z=0$ GCS masses that are consistent with observations, though no physical mechanism was proposed for the formation of GCs in this way.

In this paper, we explore a new formation channel for MP GCs directly in the halos of massive galaxies at $z \sim 6$. This is similar in spirit to the second class of models described above, but motivated by our new understanding of gas accretion and the structure of the circumgalactic medium (CGM) in massive galaxies at high redshift. Such galaxies are predicted to be fed by narrow, dense streams of cold, metal poor gas (\$2). We propose that these streams can become gravitationally unstable, leading to the formation of massive star-forming clumps in the halos of such galaxies, and in certain cases to the formation of MP GCs.

The remainder of this paper is organised as follows. In $\$ 2$ we review some of the theoretical background and observational evidence for filamentary accretion at high redshift. In \$3 we use a cosmological simulation to illustrate that streams can form bound, star-forming clumps not associated with a merging dark-matter halo, and discuss some of their properties. We then discuss recent observations that are suggestive of such stream fragmentation. In \$4 we discuss stream fragmentation analytically. We begin by estimating the characteristic radii, densities, and turbulent Mach numbers of streams as a function of halo mass and redshift. We then explore whether the streams are gravitationally unstable, and whether they can cool and form stars before reaching the 
central galaxy. Finally, we speculate when this may lead to GC formation. In $\$ 5$ we summarize our model and present specific predictions regarding the properties of MP GCs and GC systems. We discuss our results and propose avenues for future work in $\$ 6$. For the model presented in $\$ 4$ as well as the halo mass histories shown in Fig. 1, we adopt cosmological parameters $\Omega_{\mathrm{m}}=0.3, \Omega_{\Lambda}=0.7, H_{0}=70 \mathrm{~km} \mathrm{~s}^{-1} \mathrm{Mpc}^{-1}$, and a Universal baryon fraction $f_{\mathrm{b}}=0.17$.

\section{FILAMENTARY ACCRETION - THEORY AND OBSERVATIONS}

In this section we review the theoretical background of, and the observational evidence for, the existence of cold streams around massive halos, their properties, and how these relate to the assumptions of our model.

The most massive haloes at any epoch lie at the nodes of the cosmic web, and are penetrated by cosmic filaments of dark matter (e.g. Bond et al. 1996; Springel et al. 2005: Dekel et al. 2009a). We refer to such halos as stream fed halos. They represent high-sigma peaks, much more massive than the Press-Schechter mass, $M_{\nu=1}$, of typical haloes at that time (Press \& Schechter 1974). A crude upper limit for the minimal mass of a stream fed halo is that of a $2-\sigma$ peak in the cosmic density field, $M_{\nu=2}$ (Dekel \& Birnboim 2006; Dekel et al. 2009a). In Fig. 1, we show both $M_{\nu=1}$ and $M_{\nu=2}$ as a function of redshift, computed using Colossus (Diemer \& Kravtsov 2015; Diemer 2015). We also show the average mass evolution of halos with different $z=0$ masses, computed by integrating equation 2 from Fakhouri et al. (2010) for the mean mass accretion rate onto halos as a function of redshift. Lower mass halos drop below $M_{\nu=2}$ at higher redshift, and thus cease being stream fed at earlier times. For example, a $10^{11} M_{\odot}$ halo at $z=0$ ceases to be stream fed at $z \sim 5$, while a $10^{13} M_{\odot}$ halo remains stream fed until $z \sim 2$.

At $z \gtrsim 2$ the cooling time in sheets and filaments is shorter than the Hubble time (Mo et al. 2005). Furthermore, in all but the most massive clusters and their progenitors, the gas flowing along these dark matter filaments is predicted to be unable to support a stable accretion shock at the filament edge (Dekel \& Birnboim 2006; Birnboim et al. 2016). Intergalactic gas that accretes onto the filaments from sheets and voids remains dense and cold, $T \sim 10^{4} \mathrm{~K}$, as it free-falls towards the filament axis and settles in a narrow dense stream. Even in halos above $M_{\text {shock }} \sim 10^{12} M_{\odot}$, which contain hot gas at the virial temperature, the gas in streams is expected to remain cold and penetrate efficiently through the hot CGM onto the central galaxy (Dekel \& Birnboim 2006).

The above analytical picture is supported by cosmological simulations (Kereš et al. 2005; Ocvirk et al.2008; Dekel et al. 2009a; Ceverino et al. 2010; Faucher-Giguère et al. 2011; van de Voort et al. 2011; Tillson et al. 2015; Nelson et al. 2016). In these simulations, cold streams with widths of a few to ten percent of the virial radius penetrate deep into the halo. Many global properties of the streams and their interaction with the CGM and with galaxies can be deduced from simulations and compared to observations, as detailed below.

Although cold streams have not been detected directly, there is mounting circumstantial observational evidence for their existence. Cosmological simulations indicate that the streams maintain roughly constant inflow velocities as they travel from the outer halo to the central galaxy (Goerdt \& Ceverino 2015). The constant velocity, as op-

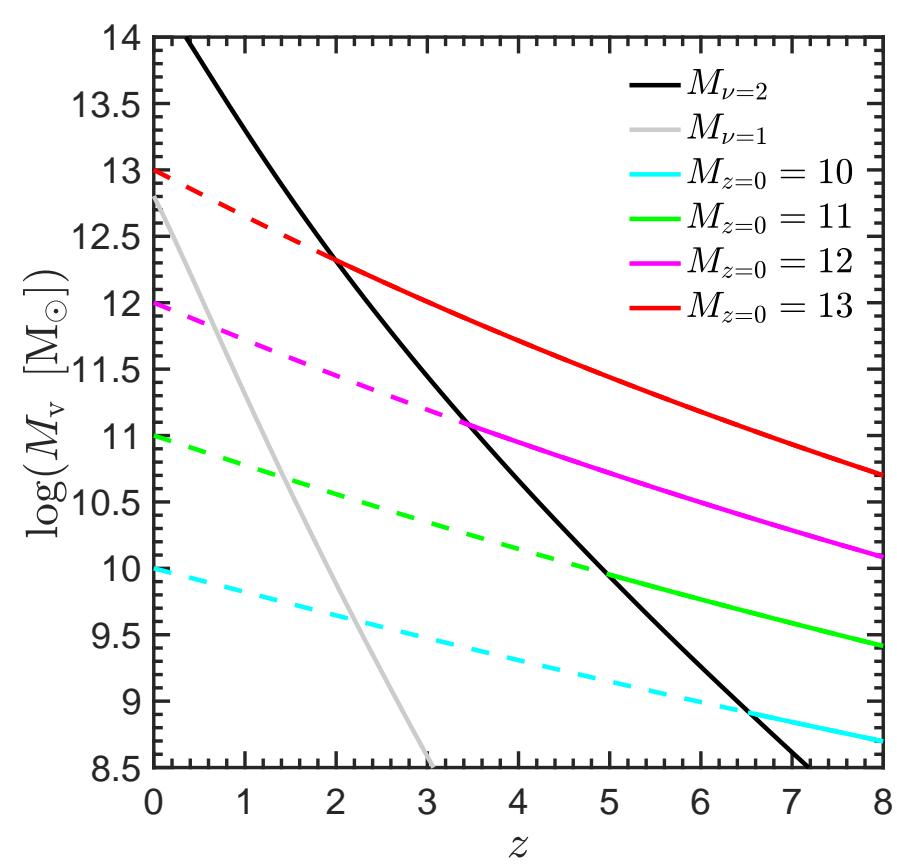

Figure 1. Redshift evolution of different halo masses. The black line shows the $2-\sigma$ mass, $M_{\nu=2}$, above which halos are likely to be stream fed. The grey line shows the Press-Schechter mass, $M_{\nu=1}$, for comparison. Colored lines show the average evolution for halos with $z=0$ halo masses $\log \left(M_{\mathrm{V}} / M_{\odot}\right)=10,11,12$, and 13 . The lines are solid when $M_{\mathrm{V}}>M_{\nu=2}$ and the halos are stream fed, and become dashed when the virial mass drops below $M_{\nu=2}$. More massive halos remain stream fed until later times.

posed to the expected gravitational acceleration, suggests energy loss into radiation which may be observed as Lyman- $\alpha$ cooling emission (Diikstra \& Loeb 2009; Goerdt et al. 2010; Faucher-Giguère et al. 2010). Radiative transfer models suggest that the total luminosity and the spatial structure of the emitted radiation appear similar to Lyman- $\alpha$ "blobs" observed at $z>2$ (Steidel et al. 2000; Matsuda et al. 2006, 2011). Radiative transfer models also show that a central quasar can power the emission by supplying seed photons which scatter inelastically within the filaments, producing Lyman- $\alpha$ cooling emission that extends to several hundred $\mathrm{kpc}$ and appears similar to observed structures (Cantalupo et al. 2014). Recent observations using the MUSE integral-field instrument suggest that such extended Lyman- $\alpha$ emitting nebulae are ubiquitous around the brightest quasars at $z \sim 3.5$ (Borisova et al. 2016; Vernet et al. 2017). The cold streams consist mostly of neutral Hydrogen and should also be visible in absorption. They can account for observed Lymanlimit systems (LLSs) and damped Lyman- $\alpha$ systems (DLAs) (Fumagalli et al. 2011; Goerdt et al. 2012; van de Voort et al. 2012). Observations using absorption features along quasar sight-lines to probe the CGM of massive SFGs at $z \sim 1-2$ reveal low-metalicity, co-planar, co-rotating accreting material (Bouché et al. 2013, 2016), providing further observational support for the cold-stream paradigm. Strong Lyman- $\alpha$ absorption has also been detected in the CGM of massive submillimeter galaxies (SMGs) at $z \sim 2$ (Fu et al. 2016).

The streams are predicted to play a key role in the buildup of angular momentum in high- $z$ disk galaxies (Pichon et al. 2011; Kimm et al. 2011; Stewart et al. 2011, 2013; Codis et al. 2012; Danovich et al. 2012, 2015). This is due both to vorticity within the filaments that spins up dark matter halos (Codis et al. 2012, 2015; Laigle et al. 2015), 

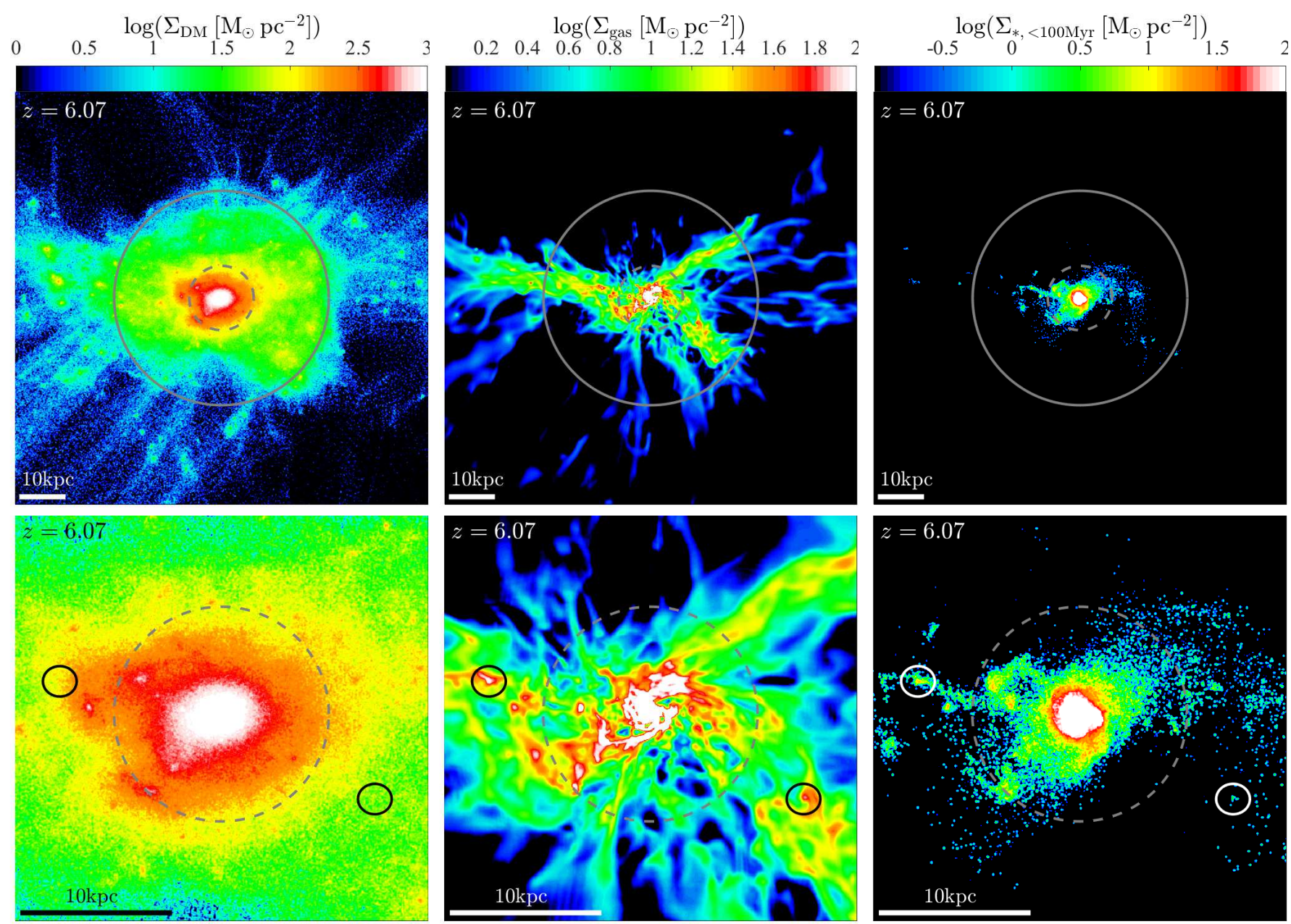

Figure 2. Filamentary accretion in a cosmological zoom-in simulation from the VELA simulation suite. We show surface densities of dark matter (left), gas (centre), and stars younger than $100 \mathrm{Myr}$ (right). The top and bottom rows are $100 \mathrm{kpc}$ and $30 \mathrm{kpc}$ on a side, respectively. Each panel is oriented face on to the central disk, and the integration depth of all panels is $40 \mathrm{kpc}$. The solid circles in the top row mark the halo virial radius, $R_{\mathrm{v}}$, while the dashed circles in the top and bottom rows mark $0.3 R_{\mathrm{v}}$. The galaxy is fed by three dense, narrow gas streams embedded within thicker dark matter filaments. These streams lie in a plane that extends beyond $R_{\mathrm{V}}$, and remain coherent outside of $\sim 0.3 R_{\mathrm{v}}$. In the inner halo, the streams can fragment into dense clumps not associated with any dark matter overdensity, where star-formation occurs. Two such clumps are circled in the bottom row. Such fragmentation can potentially lead to the formation of MP GCs far from the centres of dark matter halos.

and also to an impact parameter of the streams with respect to the galaxy centre, typically $\sim 0.3 R_{\mathrm{v}}$ (Kereš \& Hernquist 2009; Danovich et al. 2015; Tillson et al. 2015). Simulations show streams that remain cold and coherent outside of $\sim 0.3 R_{\mathrm{v}}$, inside of which a messy interaction region is seen, with strong shocks and highly turbulent flow, where the streams collide, fragment, and experience strong torques before spiralling onto the disk in an extended ring-like structure (Ceverino et al. 2010; Danovich et al. 2015). Observations of the inner regions of massive, $M_{\mathrm{v}}>10^{12} M_{\odot}$, halos at $z \sim 3$ performed with the Cosmic Web Imager (CWI) have revealed extended gaseous structures with large angular momentum (Martin et al. 2014a b). While only a handful of such cases have been observed thus far, their structure and kinematics appear very simiar to predictions from cosmological simulations of the kinematics of cold streams (Danovich et al. 2015). Similar kinematic features have been detected in absorption studies of the CGM of massive SFGs at $z \sim 1-2$ (Bouché et al. 2013, 2016).

In order to illustrate some of the concepts discussed above, we show in Fig. 2] a snapshot of a cosmological zoom-in simulation from the VELA simulation suite (Ceverino et al. 2014; Zolotov et al. 2015). The simulation was run with the adaptive-mesh refinement (AMR) code ART (Kravtsov et al. 1997; Kravtsov 2003; Ceverino \& Klypin 2009). The code incorporates gas and metal cooling, UV-background photoionization with self-shielding in gas with Hydrogen number densities $n>0.1 \mathrm{~cm}^{-3}$, stochastic star formation, gas recycling, stellar winds and metal enrichment, thermal feedback from supernovae (Ceverino et al. 2010) and feedback from radiation pressure (Ceverino et al. 2014). The grid is refined using a quasi-Lagrangian strategy based on the total mass within a cell, up to a maximal resolution of $17-35 \mathrm{pc}$ (physical) at all times, though the resolution in the outer halo can be significantly lower. Details regarding the simulation method and its limitations can be found in Mandelker et al. (2017). In Fig. 2 we show galaxy V19 (see table 1 of Mandelker et al. 2017) at $z=6.07$. At this time, V19 has a virial mass of $\sim 1.7 \times 10^{11} M_{\odot}$, a virial radius of $\sim 26 \mathrm{kpc}$, and a stellar mass within $0.1 R_{\mathrm{v}}$ of $\sim 7.8 \times 10^{9} M_{\odot}$. The last significant merger was a $\sim 1: 4$ merger at $z \sim 8$, roughly 300 Myr before the snapshot shown. 


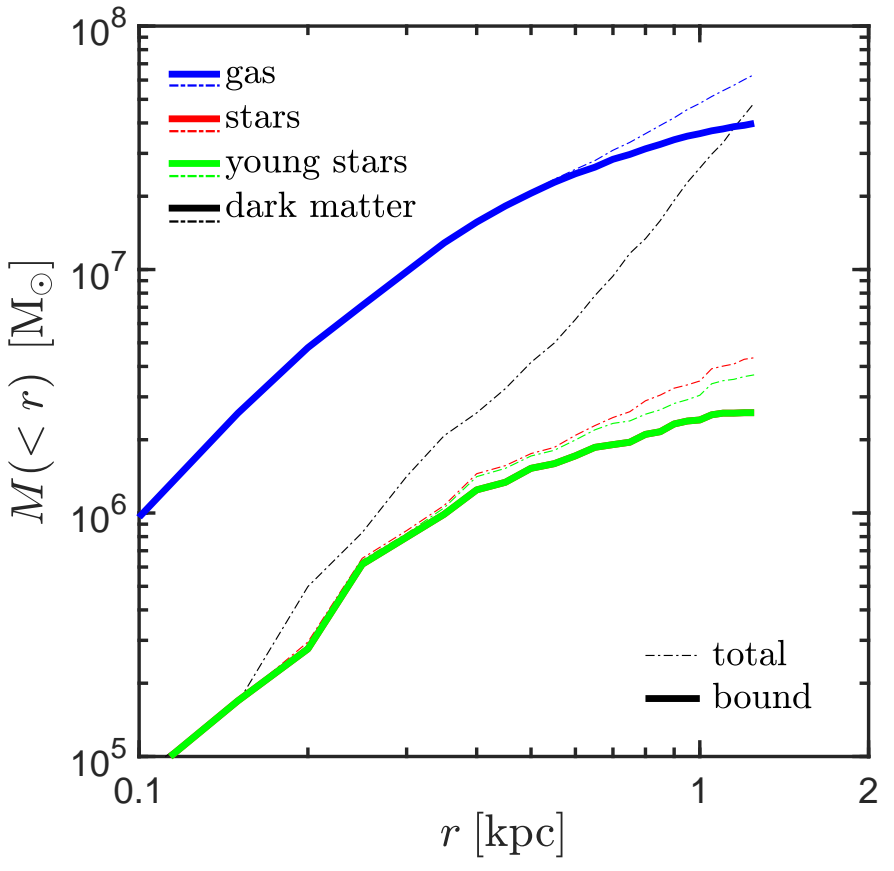

Figure 3. Mass profiles for the gas (blue), stars (red), young stars (age $<$ $100 \mathrm{Myr}$, green) and dark matter (black) in the left-hand clump marked in Fig. 2] The profiles are truncated at the tidal radius of the clump, $r_{\text {tid }} \sim$ $1.4 \mathrm{kpc}$. Thin dot-dashed lines show the total mass profiles while solid lines show the bound mass. Note that all the bound stars are young stars, so the solid green line lies on top of the solid red line. No dark matter is bound to the clump, while $\gtrsim 70 \%$ of the gas and stars are bound. Within the half mass radius of $r_{\mathrm{h}} \sim 450 \mathrm{pc}$, all of the gas and $\gtrsim 90 \%$ of the stars are bound. The mean Hydrogen number density in the inner $100 \mathrm{pc}$ is $\sim 7.1 \mathrm{~cm}^{-3}$, while within $r_{\mathrm{h}}$ it is $\sim 1.6 \mathrm{~cm}^{-3}$.

In Fig. 2, we show maps of the surface density of dark matter (left), gas (centre), and stars younger than $100 \mathrm{Myr}$ (right). The panels in the top and bottom rows are $100 \mathrm{kpc}$ and $30 \mathrm{kpc}$ across respectively. Concentric solid and dashed circles mark $R_{\mathrm{v}}$ and $0.3 R_{\mathrm{v}}$ respectively. The integration depth of all panels is $40 \mathrm{kpc}$, and they are oriented perpendicular to the angular momentum of the central star-forming disk (see Mandelker et al. 2017 for details on defining this plane). There are three prominent gas streams extending beyond the halo virial radius which seem to lie in a plane, the "stream plane". While the existence of such a plane is a generic feature in cosmological simulations, it does not typically coincide with the plane defined by the disk angular momentum as it does in this case (Danovich et al. 2012). These gas streams lie at the centres of much wider dark matter filaments. The dark matter filaments are difficult to detect within the virial radius, while the gas streams remain prominent and coherent until reaching the interaction region at $\sim 0.3 R_{\mathrm{v}}$.

\section{FRAGMENTATION AND STAR-FORMATION IN STREAMS}

\subsection{Stream Fragmentation in Cosmological Simulations}

Several previous studies have addressed the possibility of clumpy accretion along streams and its potential effects on galaxy formation and disc instability (e.g Dekel et al. 2009a b; Genel et al. 2012; Goerdt et al. 2015). Other studies addressed the possibility of clumps forming due to thermal instabilities in massive halos or in streams and its potential effect on heating the CGM in proto-clusters (Dekel \& Birnboim
2008; Birnboim \& Dekel 2011). However, the formation of baryonic clumps within streams that are not associated with dark matter halos has not been studied in cosmological simulations (though Pallottini et al. 2017 do show one such example). In Fig. 2 there are several dense clumps in the gas streams in the inner $\sim 0.5 R_{\mathrm{V}}$ that do not appear to be associated with dark matter sub-halos. Two such clumps are highlighted with circles in the bottom panels. These clumps are forming stars despite not being associated with any dark matter overdensities.

In order to verify to what extent the highlighted clumps are devoid of dark matter, we show in Fig. 3 the cumulative mass profiles of gas, stars, young stars (age $<100 \mathrm{Myr}$ ), and dark matter of the left-hand clump (hereafter clump 1) in Fig. 2 The corresponding profiles for the right-hand clump (hereafter clump 2) are qualitatively similar. The profiles are centered on the peak of gas density and extend out to the tidal radius of the clump. The tidal radius of a clump falling on a radial trajectory into a massive halo is given by the implicit formula (Tormen et al. 1998)

$$
r_{\text {tid }}=R_{\mathrm{c}}\left(\frac{m_{\mathrm{c}}\left(r_{\mathrm{tid}}\right)}{M_{\mathrm{h}}\left(R_{\mathrm{c}}\right)} \frac{1}{2-d \log M_{\mathrm{h}}\left(R_{\mathrm{c}}\right) / d \log R}\right)^{1 / 3},
$$

where $m_{\mathrm{c}}(r)$ is the total mass of the clump interior to the clumpcentric radius $r, R_{\mathrm{c}}$ is the distance of the clump from the center of the host halo, and $M_{\mathrm{h}}(R)$ is the total mass of the host halo interior to the radius $R$. The deprojected distance of clump 1 from the halo center is $R_{\mathrm{c}} \simeq 13 \mathrm{kpc} \sim 0.5 R_{\mathrm{v}}$, and its tidal radius is $r_{\mathrm{tid}} \simeq 1.4 \mathrm{kpc}$. The total mass profiles interior to $r_{\text {tid }}$ are shown in Fig. 3 as thin dot-dashed lines. We then estimate for each gas cell, stellar and dark matter particle within $r_{\text {tid }}$ whether or not it is bound to the clump, by comparing its velocity to the escape velocity from the cell/particle position to the tidal radius,

$$
V_{\mathrm{esc}}(r)=\left(2 \int_{r}^{r_{\mathrm{tid}}} \frac{G m_{\mathrm{c}}(r)}{r^{2}} d r\right)^{1 / 2} .
$$

The profiles of bound mass are shown in Fig. 3 as solid lines. There is no dark matter bound to the clump. The total mass profile of dark matter roughly scales as $m_{\mathrm{dm}}(r) \propto r^{3}$, indicating a constant background of dark matter from the host halo. On the other hand, over $70 \%$ of the gas and stellar mass interior to $r_{\text {tid }}$ is bound. The total bound baryonic mass of the clump is $\sim 5 \times 10^{7} M_{\odot}$ and its half mass radius is $\sim 450 \mathrm{pc}$. Within this radius, all of the gas and over $90 \%$ of the stars are bound, and the mean density is $\sim 2 \mathrm{~cm}^{-3}$. The mass-weighted mean stellar age of the clump is $\sim 19 \mathrm{Myr}$, yielding an average SFR of $\gtrsim 0.2 M_{\odot} \mathrm{yr}^{-1}$.

In Fig. 4 we show the mass-weighted metalicity of the gas phase in the same projection as the bottom panels of Fig. 2 Within the cold streams the typical metalicities are $[\mathrm{Z} / \mathrm{H}] \sim$ -1.5 , consistent with observed metalicities of MP GCs. Similarly low metalicities have been found in the simulations analyzed by Fumagalli et al. (2011); van de Voort \& Schaye (2012); Ceverino et al. (2016). The mass-weighted mean stellar metalicity within clump 1 is $\sim 0.02 Z_{\odot}$.

Clump 2 is qualitatively similar to clump 1 . It has a tidal radius of $\sim 850 \mathrm{pc}$, within which all the dark matter is unbound, while $>80 \%$ of the gas and stars are bound. Its gas mass is $\sim 2 \times 10^{7} M_{\odot}$, similar to clump 1 , but its stellar mass is only $\sim 2 \times 10^{5} M_{\odot}$. Its mean stellar age is $10 \mathrm{Myr}$, younger than clump 1 , with a mean stellar metalicitiy of $\sim 0.025 Z_{\odot}$. 
We stress that we are not claiming that these specific clumps represent MP GCs, which we have no hope of resolving in these simulations. In fact these clumps disrupt as they approach the central galaxy, depositing some of their stars in the halo and some of them in the disc. However, these examples highlight the fact that gas streams may become unstable and form stars and stellar clusters far from the centre of any dark matter halo. Beyond the origin of MP GCs, this can have implications for the build-up of stellar halos around massive galaxies, and for the frequency of Ly $\alpha$ emitters around massive galaxies at high redshift (Farina et al. 2017).

\subsection{Observational Evidence}

There is preliminary observational evidence for the fragmentation of cold streams. Radiative transfer models that attempt to reproduce the emission spectra of extended Lyman- $\alpha$ nebulae around luminous quasars at $z \sim 3-4$ in halos of mass $M_{\mathrm{v}} \sim 10^{12}-10^{13} M_{\odot}$, indicate that a large amount of gas is distributed in dense, compact clumps out to distances of at least $\sim 50 \mathrm{kpc}$ (Cantalupo et al. 2014; Arrigoni Battaia et al. 2015; Borisova et al. 2016). Assuming that the emission is powered mainly by photoionization from the central quasar, the models place limits on the clump properties, suggesting densities of $n>3 \mathrm{~cm}^{-3}$, sizes $R<20 \mathrm{pc}$, temperatures $T \sim 10^{4} \mathrm{~K}$, metalicities $Z<0.1 Z_{\odot}$, and typical velocities of $V>500 \mathrm{kms}^{-1}$. While such clumps would be unresolved in cosmological simulations, we note that the mean density found in clump 1 (Fig. 3) is of the same order. Under these conditions, hydrodynamic instabilities induced by the motion of these clouds through the hot halo gas should disrupt them on very short timescales (Arrigoni Battaia et al. 2015). This tension may be alleviated if the clumps are not travelling within a hydrostatic hot halo, but rather within an inflowing cold stream. It has been argued that such clumps may originate from Kelvin Helmholtz Instabilities (KHI) (Mandelker et al. 2016; Padnos et al. 2018), Rayleigh Taylor Instabilities (RTI) (Kereš \& Hernquist 2009), or thermal instabilities (Cornuault et al. 2016). Alternatively, they may be due to turbulent fragmentation in the streams (\$4.2).

Several recent observations highlight the possibility of starformation occuring inside cold streams. Recent MUSE observations of a $z \sim 6.6$ QSO, hosting a $\sim 10^{9} M_{\odot}$ black-hole with an Eddington ratio of $\sim 0.7$, have revealed the presence of a $\operatorname{Ly} \alpha$ nebula out to distances of $\sim 10 \mathrm{kpc}$ from the QSO (Farina et al. 2017). This is $\sim 25 \%$ of the virial radius of a $10^{12} M_{\odot}$ halo at $z=6.6$ (eq.7). The inferred hydrogen volume density in the nebula is $n_{\mathrm{H}} \sim 6 \mathrm{~cm}^{-3}$, similar to the densities in streams near $\sim 0.25 R_{\mathrm{v}}$ at $z \sim 6.6$ predicted by our model (eq. 24). Furthermore, the authors detected a Ly $\alpha$ emitter (LAE) at a projected distance of $12.5 \mathrm{kpc}$ from the QSO, with a line-of-sight velocity difference of $\sim 560 \mathrm{~km} \mathrm{~s}^{-1}$, comparable to the virial velocity of a $10^{12} M_{\odot}$ halo at $z=6.6$ (eq. 8), located along the direction of the extended nebula. The inferred SFR in this LAE is $\sim 1.3 M_{\odot} \mathrm{yr}^{-1}$. Based on the QSO-galaxy cross-correlation function, the authors estimate that the probability of finding such a close LAE is $<10 \%$. However, such a structure is a natural outcome of our model of star-forming clumps in gravitationally unstable streams, as detailed below. A similar example has been observed in an extended LAE near a bright QSO at $z \sim 3$ (Rauch et al. 2013), where it was speculated that feedback from the central QSO triggered a burst of star formation at an inferred rate of $\sim 7 M_{\odot} \mathrm{yr}^{-1}$ in a

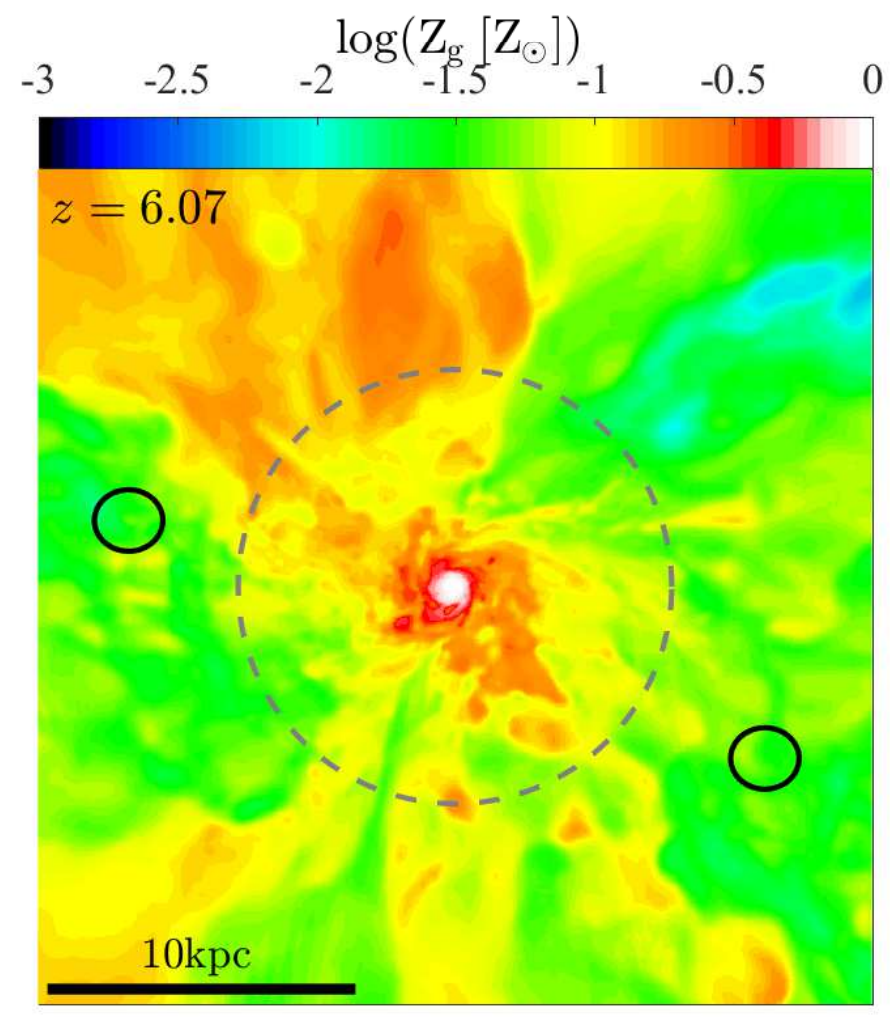

Figure 4. Metalicity distribution in the gas streams and central halo. We show the mass-weighted average gas metalicity along the line of sight, with the same projection and integration depth as in the bottom row of Fig. 2 The gas streams feeding the central galaxy have typical metalicity values of $\sim 0.01-0.03 Z_{\odot}$. The clumps forming within the streams have similar metalicity values. The high metalicity material outside the streams is low density outflowing gas driven by stellar feedback in the central galaxy.

nearby dwarf galaxy roughly $17 \mathrm{kpc}$ away from the QSO. We posit that such bursts of SF may occur directly in the dense, cold gas accreting onto galaxies without the need for a satellite dark matter halo or the "trigger" from a QSO.

Finally, ALMA observations of a very massive, $M_{*} \sim$ $2 \times 10^{10} M_{\odot}$, galaxy at $z \sim 3.5$ reveal a large structure of molecular gas extending out to $\sim 40 \mathrm{kpc}$ from the galaxy center (Ginolfi et al. 2017). This structure has a gas mass of $\sim 2-6 \times 10^{11}$, about $60 \%$ of which does not appear to be associated with either the central galaxy or its satellites. Such a large molecular gas mass cannot be accounted for by tidal stripping of satellite galaxies, and kinematic analysis does not reveal coherent rotation in the extended structure. This extended structure is also detected in continuum thermal emission, with an inferred SFR of $\sim 0.1 M_{\odot} \mathrm{yr}^{-1}$. The authors further detect gas rich systems on scales of up to $500 \mathrm{kpc}$ oriented along the same direction as the extended molecular source, with gas masses in the range $10^{10}-10^{11} M_{\odot}$ and SFRs of $30-120 M_{\odot} \mathrm{yr}^{-1}$. The authors interpret these results as gravitational collapse and fragmentation leading to starformation in the dense inner part of a cold stream feeding the central galaxy. A similar finding was reported in the Spiderweb galaxy, a massive proto-cluster at $z \sim 2.2$ observed with the Australia Telescope Compact Array (ATCA) and the VLA (Emonts et al. 2016). These authors detected $\sim 2 \times 10^{11} M_{\odot}$. of star-forming molecular gas within an extended Ly- $\alpha$ halo up to distances of $\sim 100 \mathrm{kpc}$ from the central galaxy. 


\section{ANALYTIC ESTIMATES OF STREAM FRAGMENTATION}

Motivated by the results of the previous section, we present in this section an analytical study of gravitational instability and fragmentation in cold streams. In \$4.1 we estimate the characteristic sizes and densities of cold streams. In \$4.2 we identify several sources of turbulence in the streams and estimate the resulting turbulent Mach numbers. In $\$ 4.3$ we assess the gravitational stability of streams and their characteristic fragmentation scales. In $\$ 4.4$ we discuss cooling below $10^{4} \mathrm{~K}$ and estimate when star-formation can occur in the collapsed clouds. Finally, in $\$ 4.5$ we combine all the previous aspects of the model to assess when it may be possible to form MP GCs in streams. In the next section, \$5 we summarize the main aspects of our model and highlight specific predictions for the properties of individual MP GCs and GC systems. A schematic illustration of our model, Fig. 7 , and a table summarizing the main model parameters, Table 1 is presented here. Throughout, we normalize our results to streams feeding a $10^{10} M_{\odot}$ halo at $z=6$, which corresponds to the main progenitor of a $\sim 10^{12} M_{\odot}$ halo at $z=0$, i.e. a Milky-Way progenitor (Fig. 1). However, our model applies to all halos with $M_{\mathrm{v}} \gtrsim M_{\nu=2}$, and we provide scalings with halo mass and redshift for all derived quantities.

\subsection{Characteristic Densities and Sizes of Cold Streams}

We assume that the mass flux along the streams, which have a cylindrical or conical shape, is constant along their length until they reach the central halo (e.g. Dekel et al. 2009a). We thus have

$$
\dot{M}_{\mathrm{s}}=\pi r_{\mathrm{s}}^{2} \rho_{\mathrm{s}} V_{\mathrm{s}}=M_{\mathrm{L}, \mathrm{s}} V_{\mathrm{s}}
$$

where $\dot{M}_{\mathrm{S}}$ is the mass flux along the stream, $r_{\mathrm{s}}$ is the stream radius, $\rho_{\mathrm{s}}$ is its density, $V_{\mathrm{s}}$ its velocity, and $M_{\mathrm{L}, \mathrm{s}}=\pi r_{\mathrm{s}}^{2} \rho_{\mathrm{s}}$ is the mass per unit length of the stream, hereafter the line-mass. As detailed below, we allow the stream density and radius to vary with radial position within the halo.

At $z \gtrsim 2$, the mean accretion rate of total mass (baryons and dark matter) onto the virial radius of a halo with mass $M_{\mathrm{v}}$ at redshift $z$ is well fit by 6 (Fakhouri et al. 2010)

$$
\dot{M}_{\mathrm{v}} \simeq 23 M_{\odot} \mathrm{yr}^{-1} M_{10}^{1.1}(1+z)_{7}^{2.5},
$$

where $M_{10}=M_{\mathrm{v}} / 10^{10} M_{\odot}$ and $(1+z)_{7}=(1+z) / 7$. The baryonic accretion rate onto the virial radius is given by multiplying eq. (4) by the universal baryon fraction, $f_{\mathrm{b}}$. At the high redshifts we are discussing we may assume that the accreted baryons are all gas. Cosmological simulations indicate that up to $\sim 50 \%$ of the accretion onto the halo is carried by one dominant stream, with typically two less prominent filaments carrying up to $\sim 10-20 \%$ each (Danovich et al. 2012). While these simulations focussed on $M_{\mathrm{v}} \sim 10^{12} M_{\odot}$ halos at $z \sim 2$, we assume that similar fractions apply in stream fed halos at all redshifts. We adopt $f_{\mathrm{s}}=1 / 3$ as our fiducial value for the fraction of the total accretion carried by a typical stream, and obtain for the gas accretion rate along such a stream

$$
\dot{M}_{\mathrm{s}} \simeq f_{\mathrm{s}} f_{\mathrm{b}} \dot{M}_{\mathrm{v}} \simeq 1.3 M_{\odot} \mathrm{yr}^{-1} f_{\mathrm{s}, 3} M_{10}^{1.1}(1+z)_{7}^{2.5},
$$

6 While eq. (4) may slightly underpredict the accretion rate at $z \gtrsim 5$ van den Bosch et al. 2014), we note that a nearly identical formula can be derived analytically in the EdS regime at $z>1$ (Dekel et al. 2013). where $f_{\mathrm{s}, 3}=f_{\mathrm{s}} /(1 / 3)$ and we have used $f_{\mathrm{b}}=0.17$. Fluctuations in the total accretion rate, namely in the normalization of eq. (4), can be absorbed into $f_{\mathrm{s}}$ yielding a plausible range of $f_{\mathrm{s}, 3} \sim 0.3-3$ (Dekel et al. 2013).

The flow velocity along the stream may be written as

$$
V_{\mathrm{s}}=\mathcal{M}_{\mathrm{v}} V_{\mathrm{v}}
$$

where $V_{\mathrm{v}}=\left(G M_{\mathrm{v}} / R_{\mathrm{v}}\right)^{1 / 2}$ is the virial velocity of the halo, and $\mathcal{M}_{\mathrm{v}}$ is an effective "Mach number", defined as the ratio of the stream velocity to the halo virial velocity. Cosmological simulations indicate that the streams maintain a roughly constant inflow velocity slightly below the virial value as they travel through the halo (Dekel et al. 2009a; Goerdt \& Ceverino 2015). We assume $\mathcal{M}_{\mathrm{v}} \sim 1$, with an uncertainty of a factor of $\sim 2$. The virial radius 7 and velocity of dark matter halos at $z>1$ are given by (e.g. Dekel et al. 2013):

$$
R_{\mathrm{v}} \simeq 10 \mathrm{kpc} M_{10}^{1 / 3}(1+z)_{7}^{-1} .
$$

and

$$
V_{\mathrm{v}} \simeq 66 \mathrm{~km} \mathrm{~s}^{-1} M_{10}^{1 / 3}(1+z)_{7}^{0.5},
$$

Eq. (3) can be combined with eqs. (5)- (8) to yield the typical line-mass of streams feeding a halo with virial mass $M_{\mathrm{v}}$ at redshift $z$ :

$$
M_{\mathrm{L}, \mathrm{s}} \simeq 1.9 \times 10^{7} M_{\odot} \mathrm{kpc}^{-1} M_{10}^{0.77}(1+z)_{7}^{2} f_{\mathrm{s}, 3} \mathcal{M}_{\mathrm{v}}{ }^{-1} .
$$

The line-mass of the host dark matter filament is given by dividing eq. (9) by the Universal baryon fraction,

$$
M_{\mathrm{L}, \mathrm{Fil}} \simeq 1.1 \times 10^{8} M_{\odot} \mathrm{kpc}^{-1} M_{10}^{0.77}(1+z)_{7}^{2} f_{\mathrm{s}, 3} \mathcal{M}_{\mathrm{v}}{ }^{-1} .
$$

The characteristic radius of dark matter filaments as a function of their line-mass and redshift may be estimated by considering the expansion, turnaround and virialization of cylindrical top-hat perturbations in an expanding matter dominated Universe. This is analogous to the spherical collapse model for dark matter halos. Using this model, Fillmore \& Goldreich (1984) derived the trajectories of collapsing cylindrical shells. They found an overdensity of $\sim 3.5$ within the cylinder at turnaround (in the spherical collapse model, the overdensity at turnaround is $\sim 5.55$ ), and that complete radial collapse of the shell occurs at roughly 2.5 times the turnaround time (as opposed to twice the turnaround time in the spherical collapse model). However, Fillmore \& Goldreich (1984) did not discuss virialization of the filament. The virial theorem per unit length for an infinite cylinder is

$$
2 T=G M_{\mathrm{L}}^{2},
$$

where $T$ is the kinetic energy per unit length and $M_{\mathrm{L}}$ is the mass per unit length Chandrasekhar \& Fermi 1953; Ostriker 1964). Together with the expression for the gravitational potential at radius $r$ outside a cylinder with line-mass $M_{\mathrm{L}}$,

$$
\Phi(r)=2 G M_{\mathrm{L}} \ln \left(r / r_{0}\right)
$$

where $r_{0}$ is a reference radius, one obtains a ratio of $\sim \exp (-0.25) \sim 0.78$ between the virial radius and the turnaround radius in the cylindrical collapse model (this ratio is 0.5 in the spherical collapse model). This yields a virial

\footnotetext{
${ }^{7}$ We define the halo virial radius as the radius of a sphere with mean density $18 \pi^{2} \simeq 180$ times the mean Universal matter density, valid at $z>1$.
} 
overdensity inside the collapsed cylinder of $\sim 40$. The mean density in a virialized filament is thus

$$
\rho_{\mathrm{v}, \text { Fil }} \simeq 40 \Omega_{\mathrm{m}} \rho_{\text {crit }}(1+z)^{3} \simeq 3.8 \times 10^{-26} \mathrm{gr} \mathrm{cm}^{-3}(1+z)_{7}^{3},
$$

where $\rho_{\text {crit }} \sim 9.2 \times 10^{-30} \mathrm{gr} \mathrm{cm}^{-3}$ is the critical density of the Universe at $z=0$. Combining this with eq. (10), yields the virial radius of a dark matter filament,

$$
r_{\mathrm{v}, \text { Fil }} \simeq 8 \mathrm{kpc} M_{10}^{0.38}(1+z)_{7}^{-0.5} f_{\mathrm{s}, 3}^{0.5} \mathcal{M}_{\mathrm{v}}^{-0.5}
$$

The resulting ratio of filament radius to halo virial radius is

$$
r_{\mathrm{v}, \text { Fil }} / R_{\mathrm{v}} \simeq 0.8 M_{10}^{0.05}(1+z)_{7}^{0.5} f_{\mathrm{s}, 3}^{0.5} \mathcal{M}_{\mathrm{v}}^{-0.5} .
$$

The relative width of filaments becomes smaller at later times, and has a very weak dependence on halo mass. For a $\sim$ $10^{11} M_{\odot}$ halo at $z \sim 6$, this is roughly consistent with the most prominent filament seen on the left hand side of Fig. 2 For a $10^{12} M_{\odot}$ halo at $z=2$, the typical ratio is $\sim 0.66$, which appears consistent with high-resolution cosmological zoom-in simulations (e.g. Danovich et al. 2015; Nelson et al. 2016).

The gas streams are significantly narrower than the dark matter filaments, since efficient cooling allows the gas to collapse towards the filament axis (Dekel \& Birnboim 2006; Birnboim et al. 2016). However, the final radius of the gas streams and the mechanism that supports them against gravity has not been studied in detail yet. We expect the collapsed stream to be supported by a combination of thermal and turbulent pressure (see below), and rotation as evidenced by vorticity in the filament (Codis et al. 2012, 2015; Laigle et al. 2015; Birnboim et al. 2016). Self-consistently modelling all these sources of support is beyond the scope of the current paper, and will require detailed simulations of stream evolution. In appendix $\$ \mathrm{~A}$, we show that assuming no rotation in the streams, equivalent to assuming that the streams are built by purely radial accretion of gas onto the centers of dark matter filaments, yields results that are inconsistent with both cosmological simulations and observations. Here, we adopt the opposite extreme and assume that the streams are largely supported by rotation, as has been suggested in the literature (e.g. Birnboim et al. 2016). This allows us to gain a crude estimate of the plausible sizes of streams, which turns out to be much more consistent with simulations and observations.

We assume that the stream radius, $r_{\mathrm{s}}$, scales with the radius of the dark matter filament via a contraction factor, $\lambda_{\mathrm{s}}$,

$$
\lambda_{\mathrm{s}} \equiv r_{\mathrm{s}} / r_{\mathrm{v}, \text { Fil }} \text {. }
$$

We further assume that the dark matter filament can be approximated as an isothermal cylinder truncated at $r_{\mathrm{v} . \text { Fil }}$. The density profile of an isothermal cylinder is (Ostriker 1964):

$$
\rho(r)=\rho_{\mathrm{c}}\left[1+\left(r / r_{\mathrm{h}}\right)^{2}\right]^{-2},
$$

where $\rho_{\mathrm{c}}$ is the central density of the filament, and $r_{\mathrm{h}}$ is its half mass radius. The filament line-mass profile is

$$
M_{\mathrm{L}}(r)=2 \pi \int_{0}^{r} \rho(r) r d r .
$$

Inserting eq. 17 yields

$$
M_{\mathrm{L}}(r)=M_{\mathrm{L}, \text { Fil }}\left[1-\left(1+\left(r / r_{\mathrm{h}}\right)^{2}\right)^{-1}\right],
$$

${ }^{8}$ Harford \& Hamilton (2011) quote a virial overdensity of $\sim 80$, which is what one would infer if one assumes that the ratio of virial radius to turnaround radius for collapsed cylinders is 0.5 , as it is for spheres. where $M_{\mathrm{L}, \text { Fil }}=\pi r_{\mathrm{h}}^{2} \rho_{\mathrm{c}}$ is the total line-mass of the filament. The associated circular velocity profile is

$$
V_{\text {circ }}(r)=V_{\infty}\left(r / r_{\mathrm{h}}\right)\left[1+\left(r / r_{\mathrm{h}}\right)^{2}\right]^{-1 / 2}
$$

with $V_{\infty}=\left(2 G M_{\mathrm{L}}\right)^{1 / 2}$ the circular velocity at $r \rightarrow \infty$. This can be related to the filament virial velocity using eq. (11), yielding $V_{\infty}=\sqrt{2} V_{\mathrm{v}, \text { Fil }}$. If we assume that the specific angular momentum of the gas, $j=r_{\mathrm{s}} V_{\text {circ }}\left(r_{\mathrm{s}}\right)$, is conserved during the gas contraction and that it is similar to that of the dark matter in the virialized filament, then

$$
\lambda_{\mathrm{s}}=\sqrt{2} \tilde{\lambda}_{\mathrm{Fil}} V_{\text {circ }}\left(r_{\mathrm{v}, \mathrm{Fil}}\right) / V_{\text {circ }}\left(r_{\mathrm{s}}\right)
$$

where $\tilde{\lambda}_{\text {Fil }}=j /\left(\sqrt{2} r_{\mathrm{v}, \text { Fil }} V_{\mathrm{v}, \text { Fil }}\right)$ is the filament spin parameter, defined here analogously to the halo spin parameter of Bullock et al. (2001). We denotel $r_{\mathrm{v}, \text { Fil }} / r_{\mathrm{h}}=\beta$, and thus $r_{\mathrm{s}} / r_{\mathrm{h}}=\beta \lambda_{\mathrm{s}}$. If $\beta \lambda_{\mathrm{s}}<<1$ eq. (21) reduces to

$$
\lambda_{\mathrm{s}} \simeq\left[2 /\left(1+\beta^{2}\right)\right]^{1 / 4} \tilde{\lambda}_{\text {Fil }}^{1 / 2}
$$

This yields $\lambda_{\mathrm{s}} / \tilde{\lambda}_{\mathrm{Fil}}^{1 / 2} \sim 1,0.8$ or 0.37 for $\beta=1,2$, or 10 respectively. Eq. (22) relates the stream contraction factor to the filament spin parameter. It has been known for some time that the spin parameter of dark matter halos has a constant average value of $\tilde{\lambda}_{\mathrm{H}} \sim 0.035$, independent of mass or time (Bullock et al. 2001). We assume that the filament spin parameter is likewise independent of mass or time, but with a smaller average value since the angular momentum of dark matter halos is predicted to originate from the combined angular momentum of the filament spin and orbit (impact parameter with respect to the halo centre) (Stewart et al. 2013; Danovich et al. 2015; Laigle et al. 2015). For $\tilde{\lambda}_{\text {Fil }} \sim 0.005-$ 0.02 and $\beta \sim 1-10$, we have $\lambda_{\mathrm{s}} \sim 0.02-0.14$. We hereafter assume as our fiducial value $\lambda_{\mathrm{s}} \sim 0$. 1 , but caution that this is highly uncertain and could easily vary by a factor of a few.

Cosmological simulations indicate that the streams assume a conical shape within the halo due to the gravitational attraction towards the halo center (e.g. Dekel et al. 2009a; van de Voort \& Schaye 2012). We thus assume $r_{\mathrm{s}} \propto R$ within the halo, where $R$ is the halocentric radius. Together with eqs. (14) and (16), this yields an expression for the stream radius,

$$
r_{\mathrm{s}}(R) \simeq 0.8 \mathrm{kpc} x_{\mathrm{v}} M_{10}^{0.38}(1+z)_{7}^{-0.5} € 1 f_{\mathrm{s}, 3}^{0.5} \mathcal{M}_{\mathrm{v}}{ }^{-0.5},
$$

where $€ 1=\lambda_{\mathrm{s}} / 0.1$ and $x_{\mathrm{V}}=\min \left(R / R_{\mathrm{V}}, 1\right)$. This is comparable, within a factor $\sim 2$, to the size of the large stream seen in Fig. 2] We note that in shock-heated halos with $M_{\mathrm{v}} \gtrsim 10^{12}$ streams with radii $r_{\mathrm{s}} \lesssim 0.05 R_{\mathrm{v}}$ are not expected to reach the central halo, since they will be shredded by KHI (Mandelker et al. 2016; Padnos et al. 2018). This yields a practical lower limit of $€ 1 \gtrsim 0.5$ in such massive halos.

Eq. (23) can be used together with eq. (9) to obtain the average Hydrogen number density in the streams 10 ,

$$
n_{\mathrm{H}, \mathrm{s}} \simeq 0.3 \mathrm{~cm}^{-3} x_{\mathrm{v}}^{-2}(1+z)_{7}^{3} \mathrm{t} 1^{-2}
$$

which is independent of halo mass. At $x_{\mathrm{v}} \sim 0.5$, the position of clump 1 in Fig. 2, this yields a density of $\sim 1.2 \mathrm{~cm}^{-3}$,

\footnotetext{
$9 \beta^{-1}$ is analogous to the concentration of spherical dark-matter halos.

${ }^{10}$ For a primordial composition of Hydrogen and Helium, the corresponding mass density in the streams is roughly $\rho_{\mathrm{s}} \simeq 2.3 \times 10^{-24} \mathrm{gr} n_{\mathrm{H}, \mathrm{s}}$.
} 
comparable to the density within the half mass radius of the clump, where all the gas is still bound (Fig. 3). When scaled to $z \sim 2$, eq. (24) yields densities that are comparable to gas densities found in streams in cosmological simulations, which are in the range $0.001-0.1 \mathrm{~cm}^{-3}$ (Goerdt et al. 2010; Faucher-Giguère et al. 2010; van de Voort \& Schave 2012).

Combining eqs. (23) and (24) we can estimate the column density in a typical stream,

$$
\begin{gathered}
N_{\mathrm{H}, \mathrm{s}}=2 r_{\mathrm{s}} n_{\mathrm{H}, \mathrm{s}} \simeq \\
1.4 \times 10^{21} \mathrm{~cm}^{-2} x_{\mathrm{v}}^{-1} M_{10}^{0.38}(1+z)_{7}^{2.5} \mathrm{Ł} 1^{-1} f_{\mathrm{s}, 3}^{0.5} \mathcal{M}_{\mathrm{v}}{ }^{-0.5} .
\end{gathered}
$$

For a $\sim 10^{11} M_{\odot}$ halo at $z \sim 6$, this corresponds to a surface mass density of $\sim 35 M_{\odot} \mathrm{pc}^{-2}$, within a factor of 2 of the typical gas surface densities in the streams in Fig. 2 Given their high column densities, the streams should be mostly self-shielded against the mean UV background radiation at all times. This has been found in simulations at $z \sim 2$ (Goerdt et al. 2010; Faucher-Giguère et al. 2010).

\subsection{Turbulence in Streams}

In this section we try to estimate the turbulent velocities and turbulent Mach numbers in the streams. The temperature of the stream gas is $T_{\mathrm{s}} \sim 10^{4} \mathrm{~K}$, near the Lyman cooling floor, (Dekel \& Birnboim 2006; Birnboim et al. 2016). The isothermal sound speed is thus

$$
c_{\mathrm{s}}=\left(\frac{k_{\mathrm{B}} T_{\mathrm{s}}}{\mu}\right)^{1 / 2} \simeq 8.2 \mathrm{~km} \mathrm{~s}^{-1} T_{4}^{0.5},
$$

where $T_{4}=T_{\mathrm{s}} / 10^{4} \mathrm{~K}, k_{\mathrm{B}}$ is Boltzmann's constant, and $\mu \sim$ $1.2 m_{\mathrm{p}}$ is the mean molecular weight, with $m_{\mathrm{p}}$ the proton mass. The chosen value for $\mu$ is valid for a nearly primordial composition of neutral gas. $T_{4}$ can vary in the range $\sim 0.8-3$ (Goerdt et al. 2010), absorbing any possible variation in $\mu$ as well.

The first source of turbulence we consider is accretion of gas onto the streams from the large-scale pancakes within which they are embedded (Zel'dovich 1970; Danovich et al. 2012), driven by the gravity of the streams and their host dark matter filaments. It is well established that such accretion generates turbulence (Klessen \& Hennebelle 2010; Heitsch 2013; Clarke et al. 2017; Heigl et al. 2017). Based on the models of Klessen \& Hennebelle (2010), Heitsch (2013) predicted the level of accretion driven turbulence in cylindrical filaments to be

$$
\sigma_{\mathrm{acc}}=\left(2 \epsilon r_{\mathrm{s}} V_{\mathrm{acc}}^{2} \frac{\dot{M}_{\mathrm{L}, \mathrm{s}}}{M_{\mathrm{L}, \mathrm{s}}}\right)^{1 / 3},
$$

where $\epsilon \sim 0.01-0.1$ is an efficiency parameter which is inversely proprtional to the density contrast between the filament and the accreting material Klessen \& Hennebelle 2010), $V_{\text {acc }}$ is the accretion velocity onto $r_{\mathrm{s}}$, and $\dot{M}_{\mathrm{L}, \mathrm{s}}$ is the mass accretion rate onto the stream.

We estimate the expected radial accretion velocities onto the cold streams by considering the free-fall velocity of a cylindrical gas shell starting from rest at the cylindrical turnaround radius, $\sim 1.3 r_{\mathrm{v}, \text { Fil }}(\$ 4.1)$. Note that even if the gas has some net rotation velocity, as argued in $\$ 4.1$, we are here only interested in the radial component. At $r>r_{\mathrm{v}, \text { Fil }}$ the gas is accelerated due to a constant line-mass $M_{\mathrm{L}, \mathrm{Fil}}$, which is the total line-mass of the filament. The radial velocity at $r_{\mathrm{v} \text {.Fil }}$ is

$$
v_{\mathrm{r}}\left(r_{\mathrm{v}, \mathrm{Fil}}\right)=2\left[G M_{\mathrm{L}, \mathrm{Fi}} \ln (1.3)\right]^{1 / 2} \simeq\left(G M_{\mathrm{L}, \mathrm{Fil}}\right)^{1 / 2}=V_{\mathrm{v}, \mathrm{Fil}},
$$

where we have used eq. (12) for the gravitational potential outside a filament with line-mass $M_{\mathrm{L}, \text { Fil }} . V_{\mathrm{v}, \text { Fil }}=\left(G M_{\mathrm{L}, \text { Fil }}\right)^{1 / 2}$ is the virial velocity of the dark matter filament (\$4.1). At $r_{\mathrm{v}, \mathrm{Fil}}>r>r_{\mathrm{S}}$, the line-mass as a function of radius is given by eq. (19). This can be used to compute the change in potential from $r_{\mathrm{v}, \text { Fil }}$ to $r_{\mathrm{s}}$ and thus the radial velocity at $r_{\mathrm{s}}$ :

$$
v_{\mathrm{r}}^{2}\left(r_{\mathrm{s}}\right)=v_{\mathrm{r}}^{2}\left(r_{\mathrm{v}, \mathrm{Fil}}\right)+2 G M_{\mathrm{L}, \mathrm{Fil}} \ln \left(\frac{1+\beta^{2}}{1+\left(\beta \lambda_{\mathrm{s}}\right)^{2}}\right),
$$

where we recall that $\beta=r_{\mathrm{v}, \text { Fil }} / r_{\mathrm{h}}$ and $\lambda_{\mathrm{s}}=r_{\mathrm{s}} / r_{\mathrm{v}, \text { Fil }}$. For our fiducial value of $\lambda_{\mathrm{s}}=0.1$ and $\beta=1-10$, we obtain $v_{\mathrm{r}}\left(r_{\mathrm{s}}\right) \sim$ $1.5-3\left(G M_{\mathrm{L}, \mathrm{Fil}}\right)^{1 / 2} \sim 2.3\left(G M_{\mathrm{L}, \mathrm{Fil}}\right)^{1 / 2}$. Inserting eq. (10) into eq. 29) yields

$$
V_{\mathrm{acc}} \simeq 51 \mathrm{~km} \mathrm{~s}^{-1} M_{10}^{0.38}(1+z)_{7} f_{\mathrm{s}, 3}^{0.5} \mathcal{M}_{\mathrm{v}}{ }^{-0.5} .
$$

It is striking that at $z \sim 6$ this is comparable to the virial velocity of the dark matter host halo, though it declines more rapidly with redshift. We note that the actual infall velocity onto the streams may be smaller that the free-fall velocity computed above if there is some dissipation mechanism acting on the gas as it flows towards the filament axis. Since $V_{\text {acc }}$ in eq. (30) is a factor $\sim 2$ larger than the virial velocity of the dark matter filament, this uncertainty should be within a factor of $\lesssim 2$ and can be absorbed into the efficiency parameter $\epsilon$, discussed below.

The accretion rate onto the stream can be obtained by taking the time derivative of eq. (9) and inserting eq. (4) and the relation $(1+z)_{7} \simeq(t / 0.95 \mathrm{Gyr})^{-2 / 3}$. The result is

$$
\begin{gathered}
\dot{M}_{\mathrm{L}, \mathrm{s}} \simeq 0.05 M_{\odot} \mathrm{kpc}^{-1} \mathrm{yr}^{-1} M_{10}^{0.77}(1+z)_{7}^{3.5} f_{\mathrm{s}, 3} \mathcal{M}_{\mathrm{v}}{ }^{-1} \\
\cdot\left[0.5+0.63 M_{10}^{0.1}(1+z)_{7}\right],
\end{gathered}
$$

where the expression in square brackets is $\simeq 1$ for the halo masses and redshifts we are considering. Dividing eq. (31) by eq. (9) yields the specific accretion rate of gas onto the stream,

$$
\frac{\dot{M_{\mathrm{L}, \mathrm{s}}}}{M_{\mathrm{L}, \mathrm{s}}} \simeq 2.8 \mathrm{Gyr}^{-1}(1+z)_{7}^{1.5} \text {. }
$$

Since $\dot{M}_{\mathrm{L}, \mathrm{s}} \sim 2 \pi r_{\mathrm{s}} V_{\text {acc }} \rho_{\text {acc }}$, where $\rho_{\text {acc }}$ is the density of the accreted gas, we may combine eqs. (23), 24), (30), and (31) to obtain the density contrast between the accreted gas and the stream gas:

$$
\frac{\rho_{\mathrm{acc}}}{\rho_{\mathrm{s}}} \simeq 0.02 x_{\mathrm{v}}^{3} \mathrm{E} 1^{3} .
$$

Inside the halo, the stream gas is $\gtrsim 100$ times denser than the accreted gas. This appears roughly consistent with a visual impression from cosmological simulations (e.g. Danovich et al. 2012, figure 7). Using numerical simulations and analytical arguments, Klessen \& Hennebelle (2010) found that the efficiency of converting the inflow kinetic energy to turbulent kinetic energy, $\epsilon$ from eq. (27), is approximately $\epsilon \sim \rho_{\text {acc }} / \rho_{\mathrm{s}}$, with an uncertainty of a factor of $\sim 3$. We adopt as our fiducial value a somewhat conservative $\epsilon=0.01$. Inserting this along with eqs. (30) and (32) into eq. (27) yields 
an estimate for the turbulent velocities driven by accretion

$$
\sigma_{\text {turb }, \text { acc }} \simeq 5.2 \mathrm{~km} \mathrm{~s}^{-1} M_{10}^{0.38}(1+z)_{7}\left(\epsilon_{1} x_{\mathrm{v}} € 1\right)^{1 / 3}\left(f_{\mathrm{s}, 3} / \mathcal{M}_{\mathrm{v}}\right)^{1 / 2},
$$

where $\epsilon_{1}=\epsilon / 0.01$. Dividing by the sound speed (eq. 26. yields the turbulent Mach number associated with accretion

$$
\mathcal{M}_{\text {turb,acc }} \simeq 0.7 M_{10}^{0.38}(1+z)_{7}\left(\epsilon_{1} x_{\mathrm{v}} € 1\right)^{1 / 3}\left(f_{\mathrm{s}, 3} /\left[\mathcal{M}_{\mathrm{v}} T_{4}\right]\right)^{1 / 2} .
$$

For our fiducial parameters at $z=6$ in the outer halo $\left(x_{\mathrm{v}}>\right.$ $0.5)$, the resulting turbulent Mach numbers for halos of mass $\log \left(M_{\mathrm{V}} / M_{\odot}\right)=10,11$, and 12 are $\sim 0.7,1.7$, and 4 respectively. Only for the most massive halos is the turbulence highly supersonic. In the inner halo, near $0.3 R_{\mathrm{v}}$, these values decrease by a factor of $\sim 0.67$. At $z \sim 2$ the turbulence is transonic for even the most massive halos.

In addition to accretion, there are several other potential sources of turbulence in the streams. As mentioned in $\$ 2$ in massive halos with $M_{\mathrm{v}} \gtrsim 10^{12} M_{\odot}$ that contain hot gas at the virial temperature, the streams are susceptible to KHI caused by their interaction with the halo gas. The KHI results in oblique shocks within the stream that drive turbulence with Mach numbers of order $\mathcal{M}_{\text {turb,KHI }} \sim 1$ (Padnos et al. 2018). In the inner halo, cold streams in hot halos may also be unstable to RTI provided they have an impact parameter with respect to the halo centre, placing them above the low density gas in the potential well (Kereš \& Hernquist 2009). Finally, cold streams in hot halos may also be thermally unstable (Cornuault et al. 2016), which can drive highly supersonic turbulence. We note that even in less massive halos, these processes may become relevant near the halo centre where hot gas ejected from the central galaxy due to feedback may form a hot corona (Sokolowska et al. 2017).

Another source of turbulence and instabilities in the streams is satellite galaxies flowing along the streams towards the central galaxy. Simulations suggest that up to $\sim 30 \%$ of the accretion into massive galaxies at $z \sim 3$ is associated with such satellite galaxies in the form of both major and minor mergers (Dekel et al.2009a). These galaxies can locally stir up the gas in the streams, due both to their gravitational influence on and relative velocity with respect to the stream gas, potentially inducing large turbulent motions. Furthermore, winds ejected from these satellite galaxies into the stream gas can cause shocks and stir up turbulence, and have a profound influence on the structure of streams (Faucher-Giguère et al. 2016).

Altogether we estimate that instabilities and feedback can drive turbulence in the streams with Mach numbers of $\sim 1$ to a few, preferentially in massive halos with $M_{\mathrm{v}}>10^{11} M_{\odot}$. When summed in quadrature to turbulent velocities driven by accretion, the turbulent Mach numbers in streams near $R_{\mathrm{v}}$ may reach $\mathcal{M}_{\text {turb,tot }} \sim 1,2$ and $4-5$ in halos of mass $10^{10}, 10^{11}$ and $10^{12} M_{\odot}$ respectively at $z \sim 6$. However, by $z=3$ these values should decrease by $\sim 50 \%$.

\subsection{Gravitational Instability and Fragmentation}

Self-gravitating filaments are unstable to local perturbations at wavelengths larger than the $3 \mathrm{~d}$ Jeans length, provided this is smaller than the radial scale of the filament, even in the presence of rotation (Freundlich et al. 2014). The Jeans length is given by

$$
L_{\mathrm{J}}=\left[\pi c_{\mathrm{eff}}^{2} /\left(G \rho_{\mathrm{s}}\right)\right]^{1 / 2},
$$

where $c_{\text {eff }}^{2}=c_{\mathrm{s}}^{2}+\sigma_{\text {turb }}^{2} / 3$ represents the combined radial support due to thermal and turbulent pressure. Based on the discussion in the previous section, we have $c_{\text {eff }} / c_{\mathrm{s}} \sim 1.1$, 1.5 , and 2.5 for streams in halos of mass $10^{10}, 10^{11}$ and $10^{12} M_{\odot}$ respectively at $z \sim 6$. At lower redshifts, the contribution of turbulent support decreases further. Therefore, in the following discussion we simply adopt $c_{\text {eff }}=c_{\mathrm{s}}$, and comment where relevant on how this may underestimate the resulting fragmentation scales. This has the advantage of examining the degree to which thermal pressure alone can support the streams against fragmentation, since streams are often modeled in the literature simply as isothermal cylinders at $T_{\mathrm{s}} \sim 10^{4} \mathrm{~K}$ (e.g. Dekel \& Birnboim 2006; Harford \& Hamilton 2011; Mandelker et al. 2016).

Using eqs. 23, (24) and (26) we may compute the ratio of the thermal Jeans length to the filament diameter,

$$
\frac{L_{\mathrm{J}}}{2 r_{\mathrm{s}}} \simeq 1.4 M_{10}^{-0.38}(1+z)_{7}^{-1} f_{\mathrm{s}, 3}^{-0.5} \mathcal{M}_{\mathrm{v}}^{0.5} .
$$

Note that this is independent of $\lambda_{\mathrm{s}}$ and of position within the halo. For a $10^{10} M_{\odot}$ halo, a stream carrying $f_{\mathrm{s}}=0.5$ of the total accretion has a thermal Jeans length equal to the stream width. For more massive halos $L_{\mathrm{J}}<2 r_{\mathrm{s}}$, which means that local density perturbations can trigger gravitational collapse within the streams. It can easily be verified that adopting the turbulent Jeans length does not change this conclusion. For streams where $L_{\mathrm{J}}<2 r_{\mathrm{s}}$, the characteristic mass of gravitationally unstable clumps is given by the Jeans mass,

$$
M_{\mathrm{J}} \sim(4 \pi / 3) \rho_{\mathrm{s}}\left(L_{\mathrm{J}} / 2\right)^{3} \simeq 5.85 \times 10^{7} M_{\odot} x_{\mathrm{v}}(1+z)_{7}^{-1.5} € 1 .
$$

Note that the thermal Jeans mass is independent of halo mass, and scales linearly with halocentric radius. At $z=6$, the turbulent Jeans mass is larger by a factor of $\sim 3$ and $\sim 15$ in streams feeding halos of mass $10^{11}$ and $10^{12} M_{\odot}$. At lower redshifts the difference between the thermal and turbulent Jeans masses becomes smaller. In appendix $\$[$ we show that the effects of the tidal fields induced by the host halo and the host darkmatter filament on the fragmentation scale are both negligible.

In order to ascertain whether such a Jeans unstable cloud will have time to collapse before the stream reaches the central galaxy, we compare the free fall time, $t_{\mathrm{ff}}=\left[3 \pi /\left(32 G \rho_{\mathrm{s}}\right)\right]^{1 / 2}$, to the inflow time from a given radius, $t_{\text {inflow }}=R / V_{\mathrm{s}}$. We find

$$
\frac{t_{\text {ff }}}{t_{\text {inflow }}} \simeq 0.6 \succeq 1 \mathcal{M}_{\mathrm{v}} .
$$

This is independent of halo mass, redshift, or position within the halo. Wherever the perturbation is seeded, it will collapse in roughly half the time it takes to reach the central galaxy.

For long wavelength perturbations, larger than the filament diameter, the above local stability criterion cannot be used, and we must instead examine the global stability of the filament. A self-gravitating isothermal filament is unstable to global axisymmetric perturbations if its line-mass is larger than a critial value $M_{\mathrm{L}, \text { crit }}=2 c_{\mathrm{s}}^{2} / G$ (e.g. Ostriker 1964; Inutsuka \& Miyama 1992). This can be thought of as a filamentary "Jeans line-mass", above which thermal pressure cannot prevent global radial collapse of the filament. The ratio of the line-mass in a typical stream to this critical value is

$$
\frac{M_{\mathrm{L}, \mathrm{s}}}{M_{\mathrm{L}, \text { crit }}} \simeq 0.6 M_{10}^{0.77}(1+z)_{7}^{2} f_{\mathrm{s}, 3} \mathcal{M}_{\mathrm{v}}^{-1} T_{4}^{-1} .
$$


At $z=6$, streams feeding halos more massive than $\sim 2 \times$ $10^{10} M_{\odot}$ are supercritical, and thus globally unstable. At $z=3$, the critical halo mass for unstable streams is $\sim$ $2 \times 10^{11} M_{\odot}$, smaller than $M_{\nu=2}$ at that redshift (Fig. 1 . The characteristic collapse time for a supercritical filament is $t_{\text {coll }} \sim 3\left(4 \pi G \rho_{\mathrm{s}}\right)^{-1 / 2}$ (Inutsuka \& Miyama 1992; Heitsch 2013). Comparing this to the inflow time we find

$$
\frac{t_{\text {coll }}}{t_{\text {inflow }}} \simeq 0.9 £ 1 \mathcal{M}_{\mathrm{v}}
$$

so the stream has time to globally fragment before reaching the central galaxy. This global instability results in the formation of dense cores separated by a few times the filament diameter (Inutsuka \& Miyama 1992; Clarke et al. 2016). These cores then proceed to fragment on the local Jeans scale discussed above (Clarke et al. 2016, 2017).

Our analysis thus suggests that most streams are supercritical with fragmentation times shorter than the halo crossing time. This is exacerbated further by the additional inwards gravitational force of the dark matter filament. This means that there must be some additional source of support in the streams which prevents catastrophic fragmentation in cosmological simulations. This could be rotation (\$4.1), turbulence (\$4.2), or artificial support caused by low resolution. However, Heitsch (2013) found that accretion-driven turbulence, which we expect to be the dominant source of accretion in most cases, can slow down the global collapse of supercritical filaments but it cannot halt it. The basic reason is that the line-mass of the stream grows faster than the resulting turbulent support, and therefore the stream remains super-critical. This has been confirmed by Clarke et al. (2017), who simulated a self-gravitating isothermal filament growing by accretion from its surroundings. They found that as long as the accretion flow itself was not highly turbulent, gravitational collapse and fragmentation occurs in two stages, first on large scales set by filamentary fragmentation, and then on small scales set by the local Jeans scale.

To summarize, we find that most streams feeding massive halos should be gravitationally unstable to both short and long wavelength perturbations. The former result in direct three dimensional collapse on the Jeans scale, of clouds with masses given by eq. (38). The latter result first in two dimensional filamentary collapse, followed by subsequent three dimensional collapse on the Jeans scale, leading to collapsed clouds with the same mass as in the former case. Both of these processes can act in less than a virial crossing time, particularly if the stream is relatively narrow with $€ 1 \sim 0.5$.

\subsection{Cooling and Star Formation}

In order for star-formation to occur in the collapsing gas clumps, they must be able to cool from the initial stream temperatures of $T_{\mathrm{s}} \sim 10^{4} \mathrm{~K}$ down to $\sim 10 \mathrm{~K}$. If the streams are indeed self shielded against the UV background then at $10^{4} \mathrm{~K}$ they are mostly neutral, as assumed above. At metalicities of $Z>10^{-3} Z_{\odot}$, and at the characteristic densities and temperatures of the streams, the dominant cooling process is emission in the [CII] 158 $\mu \mathrm{m}$ line (Krumholz 2012; Pallottini et al. 2017). For a cloud of gas with a mean Hydrogen number density $\bar{n}_{\mathrm{H}}=n_{0} \mathrm{~cm}^{-3}$, metalicity $Z=0.01 Z_{\odot} Z_{2}$, temperature $T=10^{4} \mathrm{~K} T_{4}$, and clumping factor $\mathcal{C}=<n_{\mathrm{H}}^{2}>/ \bar{n}_{\mathrm{H}}^{2}=10 \mathcal{C}_{10}$, the ratio of the cooling time to the free-fall time of the cloud is (Krumholz 2012, equation 6)

$$
\frac{t_{\mathrm{cool}}}{t_{\mathrm{ff}}} \simeq 9.1 \exp \left(0.009 / T_{4}\right) Z_{2}^{-1} \mathcal{C}_{10}^{-1} n_{0}^{-1 / 2} T_{4}
$$

For a collapsing cloud, this ratio decreases as the density increases during the collapse. However, since both the free-fall time and the cooling time are dominated by their initial stages near the onset of collapse, this initial ratio is representative of the final ratio. Inserting eq. (24) for the mean density in the streams and using $T_{4} \sim 1$ this yields

$$
\frac{t_{\text {cool }}}{t_{\mathrm{ff}}} \simeq 16.8 Z_{2}^{-1} \mathcal{C}_{10}^{-1} T_{4} x_{\mathrm{v}}(1+z)_{7}^{-1.5} € 1
$$

Note that this depends on position in the halo through the stream density.

Multiplying eq. (43) by eq. 39) yields the ratio of cooling time in the streams to the inflow time,

$$
\frac{t_{\text {cool }}}{t_{\text {inflow }}} \simeq 10.1 Z_{2}^{-1} \mathcal{C}_{10}^{-1} T_{4} x_{\mathrm{v}}(1+z)_{7}^{-1.5} € 1^{2} \mathcal{M}_{\mathrm{v}}
$$

This ratio increases towards lower redshifts, as the streams become less dense. We define the cooling redshift, $z_{\mathrm{cool}}$ as the redshift where the cooling time is equal to the inflow time,

$$
1+z_{\text {cool }} \simeq 32.7\left(Z_{2} \mathcal{C}_{10}\right)^{-2 / 3}\left(T_{4} \mathcal{M}_{\mathrm{v}} € 1^{2}\right)^{2 / 3} x_{\mathrm{v}}^{2 / 3} .
$$

At $z<z_{\text {cool }}$, the cooling time in the collapsing clump is longer than the inflow time to the halo center, and we do not expect much star-formation in the clump. However, at $z>z_{\text {cool }}$, the clump may experience a burst of star-formation before reaching the central galaxy.

Clumping factors of $\mathcal{C} \sim 5-10$ are not unreasonable at $z \sim 6$ given the levels of turbulence and substructure in the streams. In fact observations suggest that the clumping factors may be even higher in very massive halos $(\$ 3.2)$. We therefore assume $\mathcal{C}_{10} \gtrsim 0.5$ at $z \sim 6$, possibly approaching $\mathcal{C}_{10} \sim 1$ for $\sim 10^{12} M_{\odot}$ halos where the turbulent velocities are very large. However, as the turbulent velocities decrease towards lower redshift the associated clumping factors may decrease as well. For a relatively narrow stream with $€ 1=0.5$ near the inner halo at $x_{\mathrm{v}}=0.3$, with a metalicity of $Z_{2}=2$ (Fig. 4) and a clumping factor of $\mathcal{C}_{10}=0.5$, this yields $z_{\text {cool }} \sim 4.8$. At $z=6$, this yields $t_{\text {cool }} \sim 2.1 t_{\text {ff }} \sim 0.6 t_{\text {inflow. }}$. In this case, the clump can cool and proceed to star-formation on a free-fall timescale, before reaching the central galaxy. Furthermore, since the initial cooling timescale is longer than the free-fall timescale of the cloud, we expect relatively large contraction factors for these clouds before the onset of star-formation. This can increase the mean densities in the clouds by considerable amounts compared to the mean densities in the streams given by eq. (24). It is also worth noting that such a cloud can cool and form stars before forming any appreciable fraction of $\mathrm{H}_{2}$ (Krumholz 2012).

In order to obtain $z_{\text {cool }} \sim 2$ in a stream with fiducial parameters, we require either larger metalicities closer to $\sim 0.1 Z_{\odot}$, or larger clumping factors. Overall, we predict star-formation to be less likely to occur in the streams outside the central galaxy at $z \lesssim 4$, unless the metalicities are $\sim 0.05-0.1 Z_{\odot}$, larger than indicated by most cosmological simulations (Fumagalli et al. 2011; van de Voort \& Schaye 2012; Ceverino et al. 2016).

In order for collapse to occur the clump must also dissipate its turbulent support. The initial clump radius is of order the stream radius (eq. 37). When accounting for turbulence in the 


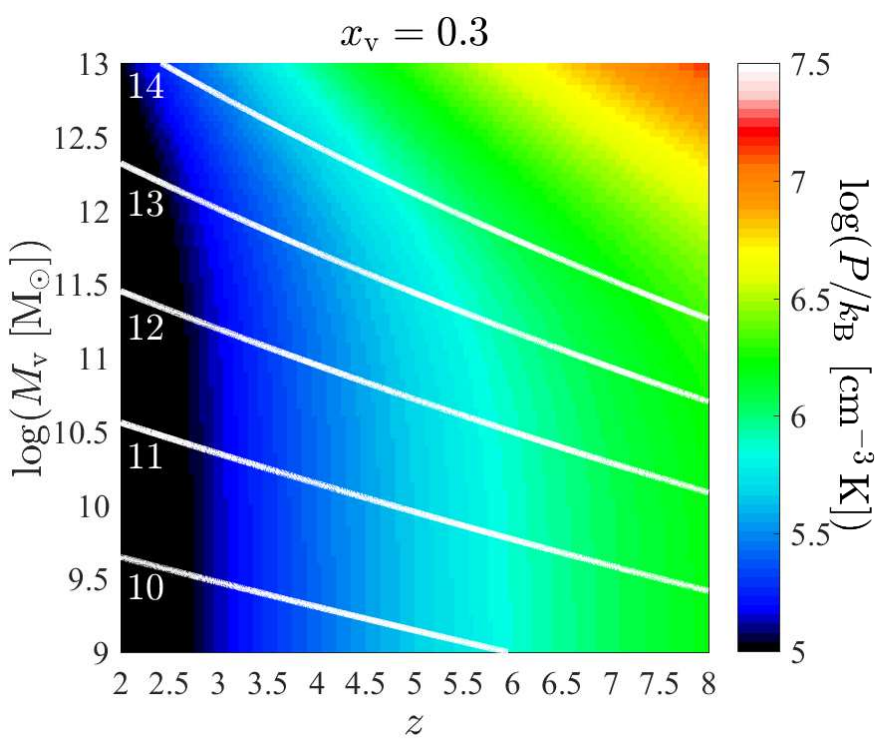

Figure 5. Average pressure, $P=\rho c_{\text {eff }}^{2}$, in the streams as a function of halo mass and redshift for parameters $f_{\mathrm{s}, 3}=\mathcal{M}_{\mathrm{v}}=T_{4}=1, \mathrm{t} 1=0.5$, and $x_{\mathrm{v}}=0.3$. This corresponds to our fiducial values for the accretion rate, velocity and temperature of the stream, but a relatively narrow stream in the inner halo at $0.3 R_{\mathrm{v}}$. The mean density is given by eq. 24, the sound speed by eq. 26, and the turbulent Mach number by eq. 35 with fiducial efficiency, $\epsilon_{1}=1$, summed in quadrature with additional Mach 1 turbulence due to instabilities and feedback. Solid white lines mark the average mass histories of halos with present day masses $\log \left(M_{\mathrm{V}}(z=0) / M_{\odot}\right)=10,11,12,13$, and 14 , as marked. At $z \gtrsim 6$, the pressure is $P / k_{\mathrm{B}} \gtrsim 10^{6} \mathrm{~cm}^{-3} \mathrm{~K}$, as required to form GCs. At lower redshifts the pressure falls below this threshold. Note that this represents the mean pressure in the stream prior to the onset of gravitational instability, while the pressure in the collapsed clouds is expected to be larger.

Jeans length, this remains true even at $M_{\mathrm{v}} \sim 10^{12} M_{\odot}$. The dissipation rate of turbulence over a length scale $r_{\mathrm{s}}$ is equal to $t_{\text {diss }} \sim r_{\mathrm{s}} / \sigma_{\text {turb }}$. Using eq. (34) as a proxy for the total turbulence in the clump, the ratio of this timescale to the inflow time can be evaluated:

$$
\frac{t_{\text {diss }}}{t_{\text {inflow }}} \simeq 0.94 x_{\mathrm{v}}^{-1 / 3} € 1^{2 / 3} \mathcal{M}_{\mathrm{v}} \epsilon_{1}^{-1 / 3}
$$

For a typical stream, the initial turbulence will dissipate in roughly the time it takes the stream to reach the central galaxy. At $x_{\mathrm{v}}=0.3$ the ratio increases to 1.4 for $€ 1=1$, but if $€ 1=0.5$ it is $\sim 0.9$.

\subsection{Formation of MP GCs}

\subsubsection{Gas Pressure in Streams}

We have established that streams at high redshift are unstable to fragmentation and that the cooling time is short enough to allow star-formation in the collapsing gas clouds. In order to form GCs in the collapsing clouds, they must reach high enough densities and pressures. GCs are expected to form only in very high pressure regions, with $P / k_{\mathrm{B}}>10^{6} \mathrm{~cm}^{-3} \mathrm{~K}$ (Elmegreen \& Efremov 1997; Kruijssen 2015). The combined thermal plus turbulent pressure in the streams prior to gravitational collapse is given by $P \simeq \rho_{\mathrm{s}} c_{\mathrm{eff}}^{2}$ (see \$4.3). This is shown as a function of halo mass and redshift in Fig. 5 We assumed fiducial values for the stream velocity and temperature, the fraction of total accretion in the stream, and the efficiency of converting accretion energy into turbulent energy. In other words, $\mathcal{M}_{\mathrm{v}}=T_{4}=f_{\mathrm{s}, 3}=\epsilon_{1}=1$. However, we assumed a relatively narrow stream, with $Ł 1=0.5$. The turbulent Mach number is given by $\mathcal{M}_{\text {turb,acc }}$ from eq. (35), summed in quadrature with an additional $\mathcal{M} \sim 1$ to account for instabilities and feedback (see \$4.2). The white lines in Fig. 5 represent mass evolution tracks of halos with $z=0$ masses $\log \left(M_{\mathrm{v}} / M_{\odot}\right)=10,11,12,13$, and 14 .

For the chosen parameters, the pressure in the streams exceeds $10^{6} \mathrm{~cm}^{-3} \mathrm{~K}$ at $z \gtrsim 6$ for all halo masses, and at $z \gtrsim 5$ for the progenitors of the most massive halos, $M_{\mathrm{v}}(z=0) \lesssim 10^{14}$. Recall that the pressure plotted in Fig. 5] which represents the pressure in the streams prior to gravitational collapse, is not enough to maintain the streams in hydrostatic equilibrium when $M_{\mathrm{L}, \mathrm{s}}>M_{\mathrm{L}, \text { crit }}$ (eq. 40). The final pressure in the collapsed clouds prior to the onset of star-formation is thus likely to be even higher. We conclude that at $z \gtrsim 6$ the pressure in the streams in the inner halo is large enough to enable formation of GCs. However, this may not be true outside of $\sim 0.3 R_{\mathrm{V}}$ due to the strong dependence of the density and pressure on position within the halo, $P \propto \rho \propto x_{\mathrm{v}}^{-2}$.

\subsubsection{Cluster Formation Efficiency}

In addition to large pressures, the formation of a GC requires very high densities. A typical GC with a mass of $2 \times 10^{5}$ and a half-mass radius of $3 \mathrm{pc}$ has a mean density within this radius of $\sim 880 M_{\odot} \mathrm{pc}^{-3} \sim 2.6 \times 10^{4} \mathrm{~cm}^{-3}$. At $z=6$, at $0.3 R_{\mathrm{v}}$, even a relatively narrow (and thus dense) stream with $€ 1=0.5$ has a typical density of $\sim 13 \mathrm{~cm}^{-3}$ according to eq. (24), roughly a factor of 2000 below the densities in GCs. However, we cannot compare the mean density in the stream or in the pre-collapse cloud to the final GC density. Star-formation in a turbulent medium is a hierarchical process where the densest objects, namely bound stellar clusters and GCs, form at the highest density peaks within the cloud (e.g. Kruijssen et al. 2012; Hopkins 2013). Furthermore, there is mounting evidence in the local Universe that the densest stellar clusters are significantly denser than the densest gas clouds (Longmore et al. 2014; Walker et al. 2015), suggesting that massive clusters are formed via hierarchical merging of smaller stellar clusters embedded in the parent gas cloud.

Within this framework, one can evaluate the fraction of star-formation occuring in bound clusters, referred to as the cluster formation efficiency, or CFE (e.g. Bastian 2008; Goddard et al. 2010), based on the density and pressure within the parent cloud 1 (Kruijssen 2012; Adamo et al. 2015; Johnson et al.2016). For a $10^{10} M_{\odot}$ halo at $z=6$ with the same parameters as in Fig. 5] the streams have pressure $P / k_{\mathrm{B}} \sim$ $10^{6} \mathrm{~cm}^{-3} \mathrm{~K}$, density $\rho \sim 0.44 M_{\odot} \mathrm{pc}^{-3}$, and column density $\Sigma \sim 100 M_{\odot} \mathrm{pc}^{-2}$. These properties are very similar to those of the Fornax model of Kruijssen (2015) (Table 1), which had $P / k_{\mathrm{B}}=1.06 \times 10^{6} \mathrm{~cm}^{-3} \mathrm{~K}, \rho=0.82 M_{\odot} \mathrm{pc}^{-3}, \Sigma=103 M_{\odot} \mathrm{pc}^{-2}$, and a CFE of $\Gamma \sim 0.38$. In our model, the density and pressure in the collapsed clouds prior to the onset of star-formation are likely to be much higher than the typical stream values, so CFE values of $\sim 0.4$ or larger are entirely plausible.

11 There is still some debate in the literature regarding the origin of the CFE, with some claiming that it does not depend on the properties of the host galaxy or of the parent cloud (Fall \& Chandar 2012; Chandar et al. 2015, 2017; Mulia et al. 2016). However, we here adopt the theoretical framework of Kruijssen (2012) whereby the CFE depends on the density and pressure in the parent cloud. 

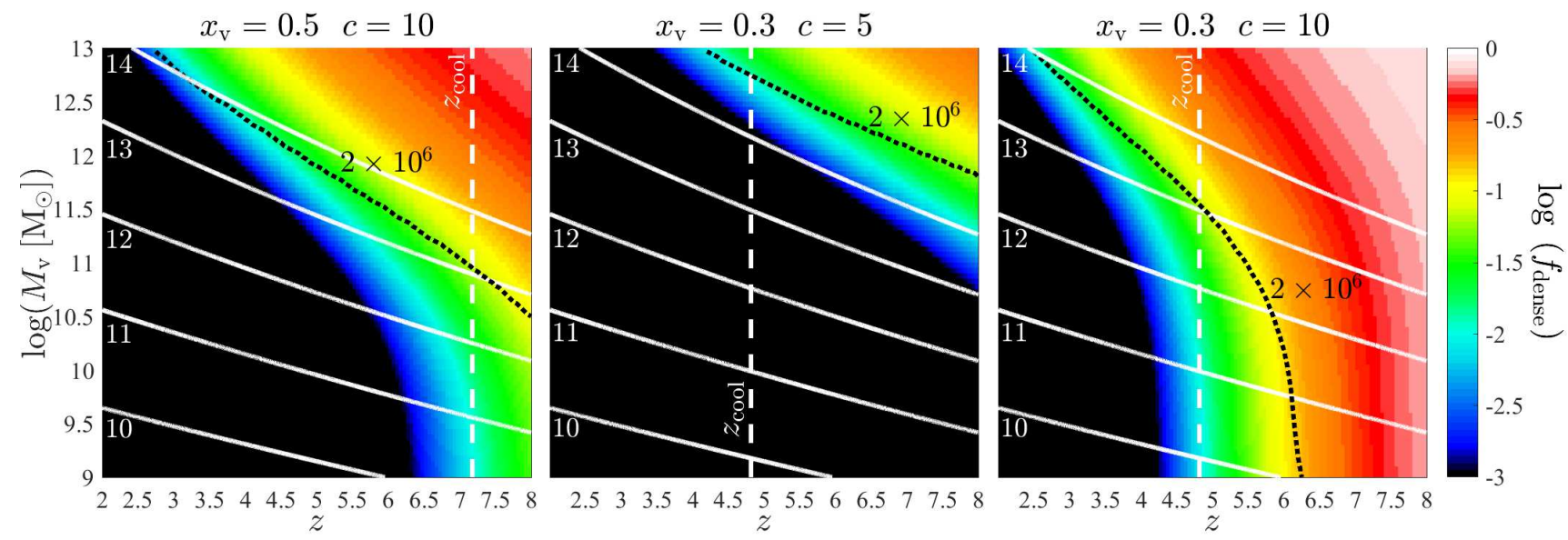

Figure 6. Mass fraction within collapsed clouds at densities $\rho>\rho_{\mathrm{GC}} \sim 880 \mathrm{M}_{\odot} \mathrm{pc}^{-3}$, high enough to form GCs. As in Fig. 5 we assume stream parameters $\mathcal{M}_{\mathrm{v}}=f_{\mathrm{s}, 3}=T_{4}=\epsilon_{1}=1$, and $€ 1=0.5$. The turbulence in the cloud is given by eq. 35) summed in quadrature with additional Mach 1 turbulence due to instabilities and feedback. The initial mean cloud density is given by eq. (24), but as the cloud contracts radially by a factor $c=5$ (center panel) or 10 (left and right hand panels) its mean density increases by a factor $c^{3}$. The turbulence induces a density distribution given by eqs. 47 and 48, with $b=0.5$. For the radial position in the halo, we show results for $x_{\mathrm{V}}=0.5$ (left hand panel) and $x_{\mathrm{V}}=0.3$ (center and right hand panels). Solid white lines mark the average mass histories of halos with present day masses $\log \left(M_{\mathrm{v}}(z=0) / M_{\odot}\right)=10,11,12,13$, and 14 , as marked. The vertical dashed lines mark $z_{\text {cool }}$ from eq. 45, the redshift above which the initial cooling time in the cloud prior to collapse is shorter than the inflow time of the stream to the central galaxy. For this equation we have assumed a clumping factor $\mathcal{C}_{10}=0.5$ and a metalicity $Z_{2}=2\left(2 \%\right.$ solar). The black dotted line in each panel marks the contour where the total mass of gas with $\rho>\rho_{\mathrm{GC}}$ is $M_{\text {dense }}=2 \times 10^{6} M_{\odot}$, assuming the total gas mass in the collapsed cloud is given by the turbulent Jeans mass, i.e. eq. 38 multiplied by a factor $\left(1+\mathcal{M}_{\text {turb }}^{2} / 3\right)^{3 / 2}$. Above this curve the clouds have enough dense gas to form GCs. Due to the relatively low stream densities in the outer halo, $x_{\mathrm{v}} \gtrsim 0.5$, the cooling time is long and we have $z_{\mathrm{cool}} \geq 7$. Furthermore, even with very large contraction factors of $c=10$ and a narrow stream, there is not enough dense gas to form GCs except at very high redshifts $z>7$. With contraction factors of $c=5$, the same is true even near the inner halo, at $x_{\mathrm{V}}=0.3$. However, at $x_{\mathrm{v}}=0.3$ with $c=10$, roughly $10 \%$ of the cloud mass is dense enough to produce GCs in halos with virial masses down to $10^{9} M_{\odot}$ at $z>5.5$, while $z_{\text {cool }} \sim 4.8$. Such large contraction factors likely require loss of angular momentum in the collapsing cloud, which can occur in the inner halo when counter-rotating streams collide (see text).

The CFE can be used to determine the maximal cluster mass that forms within the parent cloud, $M_{\mathrm{cl}, \max } \simeq \Gamma \epsilon_{\mathrm{SF}} M_{\mathrm{J}}$ where $\epsilon_{\mathrm{SF}}$ is the fraction of the cloud mass that turns into stars (Reina-Campos \& Kruijssen 2017). For $\Gamma \sim 0.3, \epsilon_{\mathrm{SF}} \sim 0.1$, and $M_{\mathrm{J}} \sim 2 \times 10^{7} M_{\odot}$ (eq. 38), we infer a maximal cluster mass of order $6 \times 10^{5} M_{\odot}$ which is again consistent with the maximal cluster mass in the Fornax model of Kruijssen (2015). For halos with $M_{\mathrm{v}}>10^{10.5} M_{\odot}$ at $z \sim 6$, which are the progenitors of $>10^{12} M_{\odot}$ halos at $z=0$, we expect larger CFE values due to stronger gravitational instability as well as larger Jeans masses due to stronger turbulence (see $\$ 4.3$ ). Both of these will yield larger cluster masses.

\subsubsection{Dense Gas Fraction}

Notwithstanding the above discussion, we defer a more detailed evaluation of the CFE and the maximal cluster mass in streams to future work, focussing here instead on the simpler question of under what conditions a collapsed cloud will have enough gas at high enough densities to lead directly to the formation of a GC. Based on the above discussion, this is a sufficient but not necessary condition for GC formation. As previously stated, a typical GC is $\sim 2000$ times denser than a typical stream. For the mean density in the collapsed cloud to reach these values, a radial contraction factor of $c=L_{\mathrm{J}} / R_{\mathrm{cl}}>13$ is required, where $R_{\mathrm{cl}}$ is the radius of the collapsed cloud. At lower redshifts and at larger halocentric distances, the discrepancy is larger. While we have argued in the previous section that the relatively long cooling times in the clouds compared to their free-fall timescale can lead to significant contraction factors before the onset of star-formation, a contraction factor of $c>10$ may still be difficult to achieve due to angular momentum support.
However, as described above it is not necessary for the mean density in the cloud to be as dense as a GC, but only that the highest density peaks are dense enough. The volumeweighted PDF of the mass overdensity in an isothermal turbulent medium is well described by a lognormal distribution (e.g. Federrath et al. 2010; Konstandin et al. 2012)

$$
d \mathrm{P} / d s=\frac{1}{\sqrt{2 \pi \sigma_{\rho}^{2}} s} \exp \left(\frac{\left(\ln (s)+0.5 \sigma_{\rho}^{2}\right)^{2}}{2 \sigma_{\rho}^{2}}\right),
$$

where $s=\rho / \bar{\rho}$ is the overdensity, and

$$
\sigma_{\rho}^{2}=\ln \left(1+b^{2} \mathcal{M}^{2}\right)
$$

where $\mathcal{M}$ is the turbulent Mach number, and $b$ depends on the ratio of compressive to solenoidal modes in the turbulence. For a "natural" mixture of modes $b \sim 0.5$, while it approaches 1 for more compressive forcing (Federrath et al. 2010). We can use this expression to estimate the fraction of mass that will be dense enough to form a GC, i.e. $\rho \gtrsim 880 M_{\odot} \mathrm{pc}^{-3}$.

We assume that the cloud begins with mean density $\rho_{\mathrm{s}}$ (eq. 24) and then contracts radially by a factor of $c$, so that its final density is $\bar{\rho} \sim c^{3} \rho_{\mathrm{s}}$. The turbulence in the initial cloud is given by $\mathcal{M}_{\text {turb,acc }}$ from eq. (35), summed in quadrature with an additional $\mathcal{M} \sim 1$ to account for instabilities and feedback, as in Fig. 5 As the cloud contracts, its internal turbulence may be amplified. In the absense of any dissipation or cooling, $\sigma_{\text {turb }}^{2} \propto G M / R$, so the turbulent Mach number scales as $\mathcal{M}_{\text {turb }} \propto c^{1 / 2}$. However, in practice this maximal enhancement is rarely seen in simulations since turbulence dissipates more rapidly as the cloud contracts. As a conservative estimate, we ignore the possible enhancement of turbulence and 
assume that it remains constant during the contraction. This may result in an underestimate of the dense gas fraction.

In Fig. 6, we show the fraction of gas in the collapsed cloud which is denser than $\rho_{\mathrm{GC}}=880 M_{\odot} \mathrm{pc}^{-3}$ as a function of halo mass and redshift. The different panels explore different values of the contraction factor, $c=5$ and 10, and the halocentric distances, $x_{\mathrm{V}}=0.3$ and 0.5 . All other parameters are the same as in Fig. 5 namely $\mathcal{M}_{\mathrm{v}}=T_{4}=f_{\mathrm{s}, 3}=\epsilon_{1}=1$ and $€ 1=0.5$. The vertical dashed line shows $z_{\text {cool }}$ from eq. (45), assuming a clumping factor of $C_{10}=0.5$ and a metalicity of $Z_{2}=2$. The white solid lines in each panel represent halo mass evolution tracks, as in Fig. 5

GCs are expected to undergo mass loss of a factor of $\sim 2-10$ from their formation until $z=0$ Kruijssen 2015; Boylan-Kolchin 2017). The formation mass of a typical GC should thus be in the range $0.5-2 \times 10^{6} M_{\odot}$. In practice, since our model predicts that GCs form directly in the halo rather than in the central disc, the actual mass loss may be on the low side (Kruijssen 2015), so a typical GC with mass $2 \times 10^{5} M_{\odot}$ at $z=0$ may have had a formation mass as low as $\sim 5 \times 10^{5} M_{\odot}$. Nevertheless, we hereafter adopt the conservative estimate that the formation mass of a GC is $\sim 2 \times 10^{6} \mathrm{M}_{\odot}$, and require at least this much gas to be above $\rho_{\mathrm{GC}}$. This can also allow for the formation of multiple clusters with a range of masses in a given parent cloud.

We can estimate the total mass of gas with $\rho>\rho_{\mathrm{GC}}$ by multiplying the fraction of dense gas shown in Fig. 6 by the turbulent Jeans mass (see $\$ 4.3$. The black dotted line in each panel of Fig. 6 marks the contour of $2 \times 10^{6} M_{\odot}$ of gas with $\rho>\rho_{\mathrm{GC}}$ in a collapsed cloud. Above this line, there is enough dense gas to form a GC. We see that in all cases, at $z>z_{\text {cool }}$ this contour roughly corresponds to the contour of $f_{\text {dense }} \sim 0.1$.

From the left-hand panel of Fig. 6 we learn that in the outer halo, at $x_{\mathrm{v}}>0.5$, even with very large contraction factors of $c=10$ only the progenitors of $\sim 10^{14} M_{\odot}$ halos at $z=0$ have enough dense gas to form GCs at $z \sim 6$. Even at $z \sim 8$, only progenitors of $\gtrsim 10^{13} M_{\odot}$ halos have enough dense gas to form GCs. We conclude that GC formation in streams is unlikely to occur in the outer halo, which was the same conclusion we reached when considering the pressure in the streams in Fig. 5

From the center panel of Fig. 6we see that even in the inner halo, at $x_{\mathrm{v}}=0.3$, a contraction factor of $c=5$ will not result in direct collapse of a GC within the collapsed cloud, except in extremely massive halos with $M_{\mathrm{v}}>10^{12.5} M_{\odot}$ at $z \gtrsim 6$.

In the right-hand panel of Fig. 6, we examine the case of $x_{\mathrm{v}}=0.3$ and $c=10$. In this case, $z_{\text {cool }} \sim 4.8$. At $z \gtrsim 5.5$, for all halos with $M_{\mathrm{v}} \gtrsim 10^{9} M_{\odot} \sim M_{\nu=2}, \sim 10 \%$ of the mass of the collapsed cloud has densities $\rho>\rho_{\mathrm{GC}}$. Furthermore, there is enough dense gas in the collapsed cloud to directly form a GC with $\sim 2 \times 10^{6} M_{\odot}$ at $z \sim 6$. We conclude that if the collapsing clouds can reach contraction factors of $c \sim 10$, then MP GC formation will occur in cold streams in the inner $\sim 30 \%$ of the halo, outside the central galaxy.

\subsubsection{Stream Collisions}

What now remains is to ask whether such a large contraction factor is possible. As noted above, since the gas stream is at least partially supported by rotation, the cloud contraction will be limited by angular momentum support at some point. While the degree of angular mometum support, and thus the maximal contraction factor, are unknown, it seems unlikely that the clumps will be able to contract in size by a factor $c=10$ without losing some angular momentum. Fortunately, there is a natural mechanism for this in the inner halo. As described in $\$ 2$ interior to $\sim 0.3 R_{\mathrm{v}}$ the streams interact with each other and with the central disc as they form an extended rotating ring and spiral towards the central galaxy within an orbital time (Danovich et al. 2015). While most of the streams are co-rotating, thus efficiently transporting angular momentum to the growing disc, simulations indicate that $\sim 30 \%$ of the mass flowing into this interaction region is actually counter-rotating (Danovich et al. 2015). A counterrotating stream can collide with another stream in this region. Such a collision could significantly reduce the angular momentum of the stream and of the collapsing clouds, as required. Furthermore, the collision itself could significantly increase the densities of the collapsing clouds. The relative velocity of the collision is of the order of the stream velocity, $\mathcal{M}_{\mathrm{v}} V_{\mathrm{v}}$, and thus has a Mach number of

$$
\mathcal{M}_{\mathrm{coll}} \simeq 8 M_{10}^{1 / 3}(1+z)_{7}^{0.5} T_{4}^{-0.5} .
$$

The very short cooling times in the streams following such a collision should result in an isothermal shock (see Cornuault et al. 2016 for a discussion on strong isothermal shocks in cold streams as they enter the virial radius of a hot halo), resulting in an increase in the stream density by a factor $\sim \mathcal{M}_{\text {coll }}^{2}$. For a $10^{12} M_{\odot}$ halo at $z=6$ this is comparable to the density increase in a cloud with a radial contraction factor of $c \sim 10$, though for lower mass halos and lower redshift the density increase is smaller. The mean density in the collapsing clouds may increase by a similar factor as well, allowing GC formation at lower global contraction factors2. We conclude that such stream collisions in the inner halo may act as the trigger for MP GC formation.

\section{SUMMARY AND PREDICTIONS OF THE MODEL}

\subsection{Stream Fragmentation}

Figure 7 presents a schematic diagram highlighting the main ingredients of our model. Massive halos at high redshift are fed by cosmic web filaments of dark matter which contain dense streams of cold, $\sim 10^{4} \mathrm{~K}$, gas in their centers. Outside the halo the filaments and streams are roughly isothermal cylinders. Inside the halo the dark matter filaments mix in with the surrounding medium and virialize, while the streams remain cold and coherent as they penetrate towards the inner $\sim 0.3 R_{\mathrm{v}}$ in a conical shape. This element of the model is not new, and has been discussed in several previous works (e.g. Danovich et al. 2015). However, our model discusses in detail for the first time gravitational instability and fragmentation of the streams. Along the way, the streams become gravitationally unstable to both large-scale cylindrical instabilities and local Jeans instabilities. They fragment into bound clumps with masses $M_{\mathrm{c}} \sim 2-5 \times 10^{7}\left(10^{8}\right) M_{\odot}$ in halos with $M_{\mathrm{v}} \lesssim 10^{11}\left(\sim 10^{12}\right) M_{\odot}$. At high redshift, typically $z>z_{\text {cool }} \sim 4$ though possibly at lower redshifts if the metalicity is more than $1-2 \%$ solar, these clumps can cool and form

12 We note that this scenario bears certain qualitative similarities to the scenario proposed by Fall \& Rees (1985), except that here the dense clouds are confined by the ram pressure of stream collisions rather than the thermal pressure of a hydrostatic halo at the virial temperature, though both these pressures have comparable magnitudes. 


\begin{tabular}{|c|c|c|c|c|}
\hline \multicolumn{5}{|c|}{ Model Parameters } \\
\hline Parameter & Meaning & Reference eqn. & Fiducial Value & Plausible Range \\
\hline 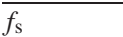 & Fraction of average halo accretion along a single stream & eq. 5 & 0.33 & $0.1-1.0$ \\
\hline $\mathcal{M}_{\mathrm{V}}$ & Ratio of stream velocity to halo virial velocity & eq. 6 & 1.0 & $0.5-2.0$ \\
\hline$\lambda_{\mathrm{s}}$ & Ratio of stream radius to dark matter filament virial radius & eq. 16 & 0.1 & $0.02-0.20$ \\
\hline$T_{\mathrm{S}}$ & Stream gas temperature & eq. 26 & $10^{4} \mathrm{~K}$ & $0.8-3 \times 10^{4} \mathrm{~K}$ \\
\hline$\epsilon$ & Turbulence driving efficiency & eq. 27. & 0.01 & $0.01-0.10$ \\
\hline $\mathcal{C}$ & Clumping factor in the streams & eq. 42 & 5 & $5-20$ \\
\hline$Z_{\mathrm{s}}$ & Stream gas metalicity & eq. 42 & 0.02 & $0.003-0.03$ \\
\hline
\end{tabular}

Table 1

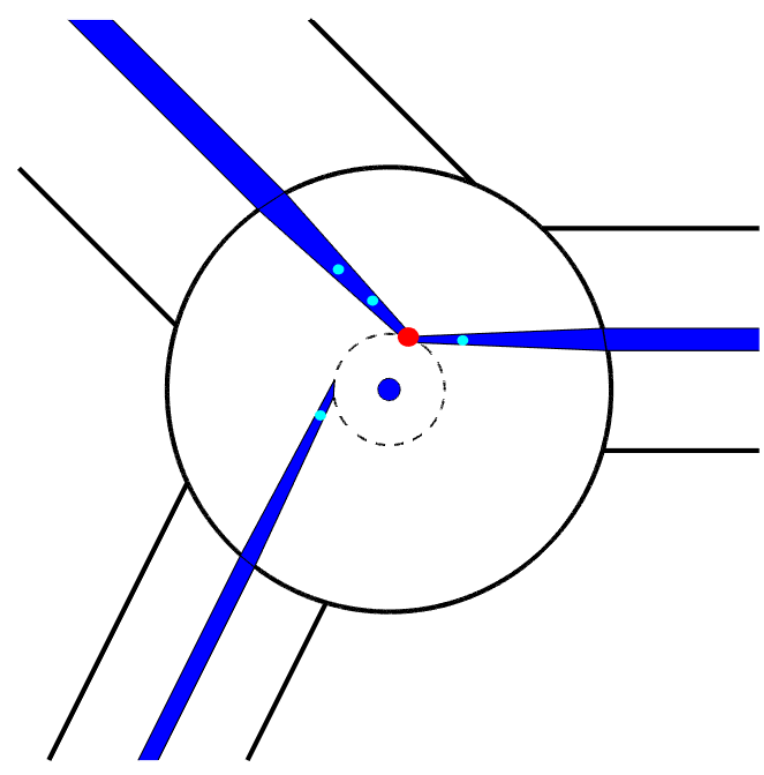

Figure 7. Schematic diagram summarizing the main components of the model. A massive dark matter halo, containing a star-forming galaxy within the inner $0.05-0.1 R_{\mathrm{V}}$, is fed by 3 cylindrical dark matter filaments with radii $r_{\mathrm{V}, \text { Fil }} \sim 0.4-0.8 R_{\mathrm{V}}$. Each dark-matter filament contains a narrow stream at its center, whose radius is roughly $r_{\mathrm{S}} \sim 0.05-0.1 r_{\mathrm{v} \text {.Fil }}$. Within the virial radius, the dark matter filaments mix in with the halo and virialize, while the gas streams form a conical shape and plunge towards the halo center. These gas streams have impact parameters of the order $\sim 0.3 R_{\mathrm{v}}$, marked by a dashed circle. Within this region the streams interact with each other and with the central disc galaxy until they eventually join the disc. Some streams may counter-rotate with respect to the total angular momentum, resulting in a stream collision within the interaction region. This will lead to the formation of a massive, bound GC. Less massive clusters and field stars can form within the streams themselves farther out in the halo.

stars before reaching the central galaxy. This is seen in cosmological simulations (Figs. 2]and 3), and may have been observed in a few instances (see $\$ 3.2$ ). Little or no dark matter is expected to be bound to these clumps despite their location at distances of up to tens of kpc from the central galaxy. While many of these clumps may eventually be destroyed by the tidal forces of the central galaxy, they can contribute to the growth of the metal-poor population in the stellar halo $\left(Z \lesssim 0.03 Z_{\odot}\right.$, Fig. 4$)$ and may be observed as Ly $\alpha$ emitters.

There are seven parameters that define the various aspects of our model. These are summarized in Table 1, along with the equation where they first appear and with their fiducial values and plausible ranges, based on the arguments laid forth in $\$ 4$. The gravitational stability of cold streams depends on $f_{\mathrm{s}}, \mathcal{M}_{\mathrm{v}}$, and $T_{\mathrm{s}}$, with faster, hotter and less prominent streams being more stable (eqs. 37 and 40. Pushing these to their extreme values, our model predicts that the minimal halo mass where streams are likely to be gravitationally unstable at $z=6$ is in the range $\sim 2 \times 10^{9}-2 \times 10^{11} M_{\odot}$, while for typical values halos with $M_{\mathrm{v}} \gtrsim 2 \times 10^{10} M_{\odot}$ at $z=6$ should host unstable streams. Whether this instability is able to manifest itself in the halo, before the streams reach the central galaxy, depends primarily on the product $\mathcal{M}_{\mathrm{v}} \lambda_{\mathrm{s}}$ (eqs. 39, 41, and 45). For typical values, clumps collapse and cool before reaching the galaxy, though if a stream is both very wide and rapidly inflowing this may not be the case. The thermal Jeans mass in the streams depends on stream width, $\lambda_{\mathrm{s}}$, and on position within the halo (eq. 38). For the full range of plausible $\lambda_{\mathrm{s}}$ values and for halocentric distances of $(0.3-1) R_{\mathrm{v}}$, the thermal Jeans mass is $\sim 4 \times 10^{6}-10^{8} M_{\odot}$, though it is expected to typically be a few times $10^{7} M_{\odot}$.

The level of turbulence in the streams depends on the first five parameters in Table 1 (eq. 35). The turbulent Mach number increases for slower, wider, colder, and more prominent streams, with a larger driving efficiency. In our fiducial model, turbulence played a significant role only in the most massive halos, $M_{\mathrm{v}} \sim 10^{12} M_{\odot}$ at $z=6$, while for less massive halos and at lower redshifts thermal pressure dominated over turbulent pressure. Therefore, if the parameters are such that the turbulence is even lower, this will not alter our main conclusions. On the other hand, if we take extreme values for all parameters to make the turbulence as large as possible $\left(f_{\mathrm{s}}=1, \mathcal{M}_{\mathrm{v}}=0.5, \lambda_{\mathrm{s}}=0.2, T_{\mathrm{s}}=0.8 \times 10^{4} \mathrm{~K}, \epsilon=0.05\right)$, the turbulent pressure can dominate even in $10{ }^{10} M_{\odot}$ halos. This would yield larger density and pressures peaks in the streams, thereby strengthening the case for globular cluster formation. However, such a parameter combination seems unlikely, and we do not expect turbulence to be significant in halos less massive than a few times $10^{11} M_{\odot}$.

Finally, the redshift above which the streams can cool to $T \lesssim 100 \mathrm{~K}$ and form stars before reaching the central galaxy depends on the product of $\mathcal{C} Z_{\mathrm{s}}$ (as well as $\mathcal{M}_{\mathrm{v}} \lambda_{\mathrm{s}}$ as described above, eq. 45). A stream with both very low metalicity and very small clumping factor will not be able to cool and form stars at $z \sim 5-6$ as in our model. However, our fiducial clumping factor of $\mathcal{C} \sim 5$ is at the low end of the expected range, and cosmological simulations suggest that streams feeding massive galaxies have been enriched to roughly $1-3 \%$ solar metalicity by $z \sim 6$, so we expect that for typical cases streams should be able to cool and form stars at $z \gtrsim 5$. The plausible range for these parameters may even lead to cooling and star-formation at somewhat lower redshifts.

\subsection{Globular Cluster Formation}

At $z \gtrsim 6$, in the inner $\sim 0.3 R_{\mathrm{v}}$ for a relatively narrow (though by no means extreme) stream with $\lambda_{\mathrm{s}} \sim 0.05$, the pressure in the streams is large enough to enable GC formation (Fig. 5). The cluster formation efficiency (CFE) is 
expected to be $\gtrsim 0.3-0.4$, yielding maximal cluster masses of $\sim 6 \times 10^{5} M_{\odot}$ in halos with $M_{\mathrm{v}} \lesssim 10^{10} M_{\odot}$. The maximal cluster mass may increase by a factor of $\gtrsim 10$ in halos with $M_{\mathrm{v}} \sim 10^{12} M_{\odot}$, both because of an increased turbulent Jeans mass and an expected increase of the CFE. These values are consistent with previous estimates of GC masses at formation in models where GCs form in dense GMCs within high redshift discs (Kruijssen 2015). The low metalicity in the cold streams results in a low metalicity of $\lesssim 0.03 Z_{\odot}$ for these clusters, similar to MP GCs. However, due to the strong dependence of the density and pressure on stream width, it is unlikely that regular star formation in the streams will result in $\mathrm{GC}$ formation for $\lambda_{\mathrm{s}} \gtrsim 0.1$.

The inner $\sim 0.3 R_{\mathrm{V}}$ consists of a messy interaction region where the streams join the disc. In this region, collisions between counter-rotating streams can result in densities and pressures large enough to form GCs even if $\lambda_{\mathrm{s}} \sim 0.1$ (Fig. 6), and may be the dominant formation channel of MP GCs in our model. These proto-GCs may have masses of $\lesssim 10 \%$ the mass of their parent cloud, namely a few times $10^{6} M_{\odot}$ $\left(10^{7} M_{\odot}\right.$ for the most massive halos), and be essentially devoid of dark matter. Their masses then decrease by a factor of $3-10$ by $z=0$, yielding masses typical of observed GCs.

Our model may allow for formation of GCs at relatively low redshifts, down to $z \sim 4.7$ for the progenitors of $10^{13} M_{\odot}$ halos at $z=0$, and down to $z \sim 4$ for the progenitors of $10^{14} M_{\odot}$ halos. The latter is assuming larger clumping factors due to larger turbulent velocities, in order to allow the collapsing clouds to cool before reaching the halo centers. This likely results in higher metalicities for the GCs formed in more massive halos, owing to the overall larger metalicities at lower redshifts. Additionally, those GCs that form at lower redshift form in more massive clouds (eq. 38). A trend between the mass of individual MP GCs and the mass of their host halo is indeed observed (Harris et al. 2017). This is also qualitatively consistent with the observed correlation between the mass and metalicity of MP GCs, the "blue tilt", since more massive halos at lower redshift will have higher metalicity gas. This feature is not observed for MR GCs (e.g. Brodie \& Strader 2006), possibly hinting at a different formation environment for the two populations.

The kinematics of the MP GC population is a result of the kinematics of the streams in the inner $\sim 0.3 R_{\mathrm{v}}$ where we estimate fragmentation is most likely to occur. As discussed in $\$ 2$, this is the region where the angular momentum is transferred from the streams to the disc due to strong torques, and the streams form an extended, high angular momentum, ring-like structure. Formation of MP GCs in these conditions may help explain the rapid rotation velocities and velocity anisotropies observed in the MP GC population in the outer halos (Pota et al. 2015a).

Our picture of MP GC formation in the inner halo allows us to place constraints on two important observables: the ratio of the mass of a MP GC system (GCS) to that of its host halo at $z=0$, and the ratio of the extent of a GCS to the virial radius of its host halo at $z=0$. Both of these are observed to be constant over several orders of magnitude in halo mass (Harris et al. 2017; Forbes 2017).

An upper limit to the total mass of the MP GCs that formed in situ in the host halo as a result of collisions between counter-rotating streams, rather than having been accreted at lower redshift, can be obtained as follows. As stated above,

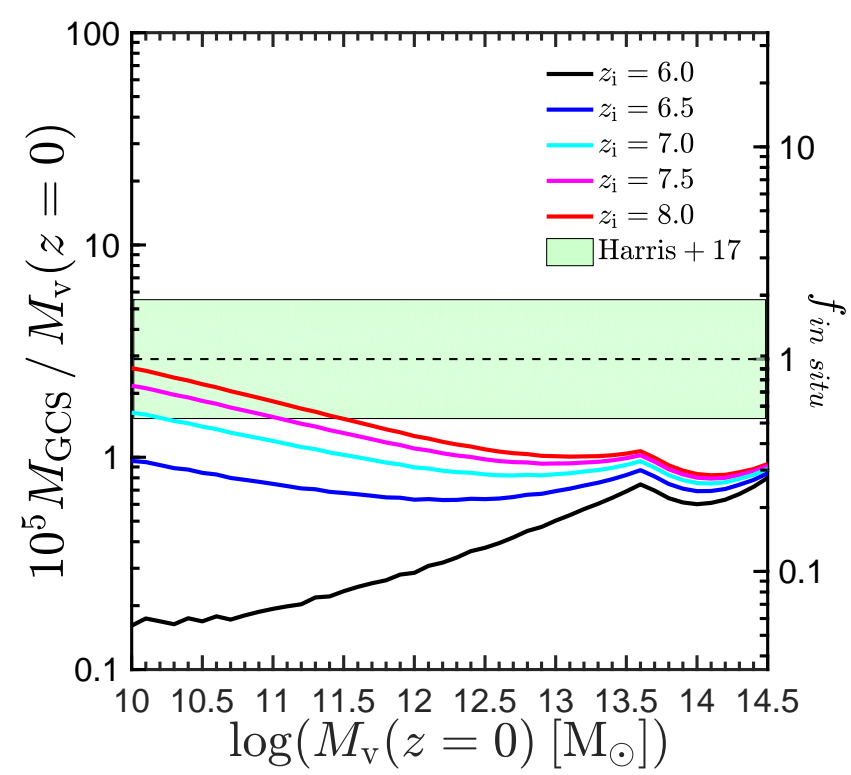

Figure 8. Estimate of the ratio of the total mass in MP GCs that formed within the halo at $z \sim 6$ to the mass of the host halo at $z=0$, as a function of host halo mass, according to our model. The solid curves represent our model, which assumes that all counter-rotating gas that enters $0.3 R_{\mathrm{V}}$ between an initial redshift $z_{\mathrm{i}}$ a final redshift $z_{10 \%}$ forms MP GCs at $10 \%$ efficiency. $z_{10 \%}$ is defined as the redshift where $f_{\text {dense }}$ from Fig. 6 falls below $10 \%$, and we have assumed the same model parameters as in the right-hand panel of Fig. 6] In halos with $M_{\mathrm{v}}<10^{12} M_{\odot}$ all gas entering the virial radius is assumed to penetrate down to $0.3 R_{\mathrm{V}}$, while in more massive halos the penetration is only $\sim 50 \%$ (Dekel et al. 2013). The counter-rotating gas represents $30 \%$ of the total gas entering $0.3 R_{\mathrm{v}}$, based on simulations (Danovich et al. 2015). The MP GCs then decrease in mass by a factor of 10 by $z=0$. Different colour lines show results for different values of $z_{\mathrm{i}}$, ranging from 6 to 8. The dashed line and shaded region show the mean ratio and $1-\sigma$ scatter from Harris et al. (2017) for the total mass of MP GCs to the host halo mass, $M_{\mathrm{GCS}} / M_{\mathrm{V}} \sim 2.9 \times 10^{-5} \pm 0.28$ dex. At low halo masses, where accretion of ex situ GCs via mergers is expected to be negligible, our model is in good agreement with the observations. At higher halo masses we predict a larger fraction of accreted GCs. For MW mass halos, $M_{\mathrm{V}} \sim 10^{12} M_{\odot}$, we predict that $\sim 30 \%$ of the MP GCs were formed in halos at $z \gtrsim 6$, in good agreement with the prediction of Boylan-Kolchin (2017). The kink in the model curves at $M_{\mathrm{V}} \sim 10^{13.5} M_{\odot}$ marks halos that become more massive than $10^{12} M_{\odot}$ at $z>z_{10 \%}$.

simulations indicate that $\sim 30 \%$ of the gas mass entering $0.3 R_{\mathrm{V}}$ is counter-rotating. We assume that all the counterrotating gas that enters $0.3 R_{\mathrm{v}}$ between an initial redshift $z_{\mathrm{i}}$ and the redshift where $f_{\text {dense }}$ from Fig. 6 falls below $10 \%, z_{10 \%}$, contributes to formation of MP GCs at $10 \%$ efficiency. At these high redshifts, the halo mass as a function of redshift is well approximated by $M_{\mathrm{v}}(z) \simeq M_{\mathrm{v}}\left(z_{0}\right) \exp \left(-0.79\left[z-z_{0}\right]\right)$ (Dekel \& Mandelker 2014). For all halos of interest, $z_{10 \%} \sim$ $5-6$. For a given $z_{\mathrm{i}}$, the fraction of mass accreted onto a halo in the redshift range $z_{\mathrm{i}}-z_{10 \%}$ is independent of halo mass. For $z_{\mathrm{i}} \sim 7-8$, this fraction is $\sim 0.55-0.90$. Thus the efficiency of GC formation relative to the total baryonic mass in the halo at $z_{10 \%}$ is $\sim 0.1 \times 0.3 \times(0.55-0.90)=0.0165-0.027$, independent of halo mass. In his empirical model of hierarchical merging of globular cluster systems, Boylan-Kolchin (2017) found that formation efficiencies of $\sim 1.4-2.8 \%$ for MP GCs in halos at $z=6$, combined with GC mass loss by a factor of $\sim 10$ until $z=0$, can account for the present day masses of GC systems.

If we assume additional mass loss by a factor of $\sim 10$ until $z=0$, we have $\sim 1 \%$ of the counter-rotating mass entering 
$0.3 R_{\mathrm{v}}$ from $z_{\mathrm{i}}$ to $z_{10 \%}$ winds up in MP GCs at $z=0$. Note that this represents mass loss from the entire GCS rather than from an individual GC, and thus includes GC disruption as well. This is $0.01 \times 0.3 \times p=0.003 p$ of the total gas mass or $0.003 p \times f_{\mathrm{b}} \sim 5.1 \times 10^{-4} p$ of the total mass accreted onto the halo in this interval, where $p$ is the fraction of gas crossing the virial radius that penetrates down to $0.3 R_{\mathrm{v}}$. In halos with virial mass $M_{\mathrm{v}} \lesssim 10^{12}$ we expect $p \sim 1$, while in more massive halos we expect $p \sim 0.5$ due to the presence of a hot halo out to the virial radius (Dekel et al.2013; Dekel \& Mandelker 2014). This is only relevant for the progenitors of very massive halos with $M_{\mathrm{v}}>10^{13.5} M_{\odot}$ at $z=0$, since lower mass halos are always less massive than $10^{12} M_{\odot}$ at $z>z_{10 \%}$ (Fig. 6).

In Fig. 8 we show the results of this simple model for different values of $z_{i}=6-8$, for the same model parameters as in the right-hand panel of Fig. 6. For each chosen $z_{\mathrm{i}}$ we show the ratio of the total GCS mass to the host halo mass at $z=0$ as a function of host halo mass. The kink at $M_{\mathrm{v}} \sim 10^{13.5}$ in all model curves is due to the progenitors of these massive halos crossing the $10^{12} M_{\odot}$ threshold at $z>z_{10 \%}$. For comparison we show the recent result of Harris et al. (2017), who found a ratio of $\sim 2.9 \times 10^{-5} \pm 0.28 \mathrm{dex}$. For $z_{\mathrm{i}} \geq 7.5$, our results for low mass halos with $M_{\mathrm{v}}<10^{11} M_{\odot}$ are in very good agreement with the observations, suggesting that a large fraction of their MP GCs could have formed via this mechanism. At higher masses we underproduce the total mass of the MP GCS. But this is to be expected, since we are only accounting here for MP GCs that formed as a result of stream collisions in the halo. The remaining GCs are ex situ in origin, accreted onto the halo during mergers of sub-halos 13 . We find that the fraction of mass in ex situ MP GCs accreted during mergers increases with halo mass, because more massive halos accumulated a larger fraction of their total mass through mergers of halos with $M_{\mathrm{v}}>10^{9} M_{\odot}$ at $z<6$, the threshold for in situ MP GC formation in our model. For a MW mass galaxy, with $M_{\mathrm{V}}(z=0) \sim 10^{12} M_{\odot}$, we find that $\sim 30 \%$ of the total GC mass was formed in the halo of the main progenitor at $z \gtrsim 6$. This is in good agreement with the estimates of Boylan-Kolchin (2017) who constrained this value using halo merger trees. In his model, he assumed that every halo above $\sim 10^{9} M_{\odot}$ at $z=6$ formed MP GCs in the halo with a total mass proportional to the halo mass at $z=6$, but did not propose a physical mechanism by which this could occur. Our model provides such a physical mechanism, and forms the basis for formation of MP GCs directly in the halos of massive galaxies at $z \sim 6$. Hierarchical models can then be used to assess the accretion of $e x$ situ GCs via mergers of sub halos.

In addition to the total mass, we can also place constraints on the radial extent of the in situ MP GCs by assuming that most MP GCs are formed at $\sim 0.3 R_{\mathrm{v}}$ at $z_{10 \%}$, and remain at the same physical distance from the halo center at $z=0$. Indeed, the dynamical friction timescale for a $10^{6} M_{\odot} \mathrm{GC}$ on a circular orbit at $0.3 R_{\mathrm{v}}$ in a $10^{10} M_{\odot}$ halo is $\sim 16 \mathrm{Gyr}$, longer than the age of the Universe (Binney \& Tremaine 2008 equation 8.13, assuming a Coloumb logarithm of $\ln (\Lambda)=10)$. Figure 9 shows the results of this simple model in comparison to recent observations by Forbes (2017). The observational data show the half-number radii of GCs in a sample of $\sim 30$

\footnotetext{
${ }^{13}$ As discussed, we also expect some MP GC formation in streams feeding massive halos even in the absense of collisions. The contribution of these clusters to the total population depends on both the CFE and the total amound of star formation in the streams, and will be the subject of future work.
}

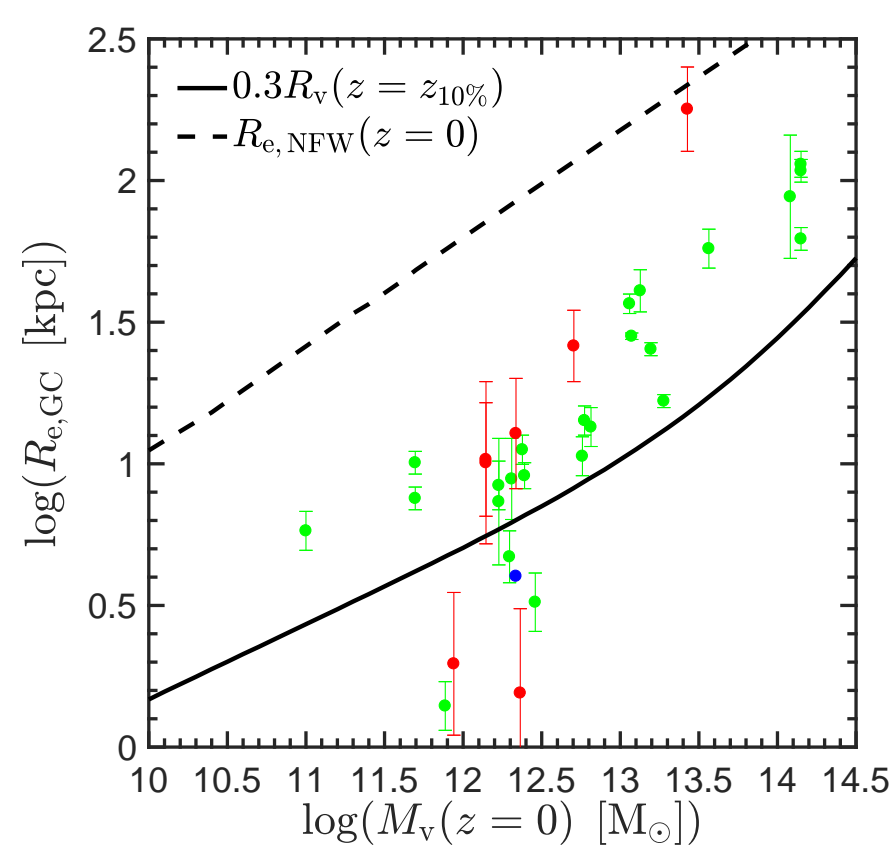

Figure 9. Radial extent of MP GCs at $z=0$ as a function of host halo mass. The points show data from Forbes (2017), specifically their figure 5. The red points are galaxies from the study of Hudson \& Robison (2017), the green points are other data from the existing literature including three UDGs, and the blue point is the MW. Mesurement data represent the half-number radius of the GCS. The solid black line shows the prediction from our model for the radial extent of those MP GCs that formed within the halos of their host galaxies at $z \sim 6$. We assume that the radial extent of these MP GCs is $\sim 30 \%$ of the virial radius of their hos halo at $z_{10 \%}$, defined as in Fig. 8 Our model systematically lies $\sim 0.1-0.3$ dex below the observations, though the slope of our predicted relation is very similar to the observed slope, including the steepening above $10^{13} M_{\odot}$. The larger observed sizes are due to additional ex situ GCs that were accreted onto the halo via mergers and lie at larger galactocentric distances. Additionally, mixing of dark matter and GCs caused by violent relaxation during halo assembly can pump up the radial extent of a GCS out to an upper limit given by the half-mass radius of the dark matter halo, shown by the dashed line. The observed data lie in between these two limits.

GCSs spanning 3 orders of magnitude in halo mass, from $M_{200} \sim 10^{11}-10^{14} M_{\odot}$, and including ultra-diffuse galaxies, giant ellipticals and the MW. This study found that the radial extent of GCs was proportional to the virial radius of their host halo, scaling as $M_{\mathrm{v}}^{1 / 3}$. However, we do note that Hudson \& Robison (2017) found a different, non-linear, scaling of the extent of GCSs with halo virial radius, albeit using a smaller sample than Forbes (2017). Further observations are needed to clarify this point. Comparing our simple model to the observations, we see that while we systematically underpredict the radial extent of GCSs by $\sim 0.1-0.3$ dex compared to the observations, the slope of the GCS size-halo mass relation is consistent with observations, including the steepening at $M_{\mathrm{v}}>10^{13} M_{\odot}$. In our model, this steepening is due to $z_{10 \%}$ decreasing below $z \sim 6$, towards $z \sim 4.8$ and 4 for $10^{13}$ and $10^{14} M_{\odot}$ halos.

The larger observed radial extents of GCSs compared to our simple model indicates that ex situ GCs which are accreted onto the halo via mergers tend to lie at larger galactocentric distances than those that formed in situ. Furthermore, violent relaxation associated with halo assembly from $z \sim 6$ to $z=0$ will mix the dark matter and GCs, possibly increasing the effective radius of the GCS. The maximal radius that can be reached by this process is the half-mass radius of the 
dark matter halo. In Fig. 9 we show the effective radius of an NFW halo as a function of halo mass, assuming the massconcentration relation of Zhao et al. (2009). The slope of this relation is similar to the observed slope, and the observed data are roughly midway between this upper limit and our estimated lower limit.

Finally, we stress that our model makes a testable prediction, namely that at high redshift during the epoch of GC formation, both MP GCs and less dense star-forming clouds should trace the streams that feed galaxies. They should reside outside galaxies, at distances of up to tens of kpc, along filamentary, possibly ring like structures. Future observations with JWST are expected to observe the formation of GCs (Renzini 2017; Boylan-Kolchin 2017), and will be able to test these predictions.

\section{DISCUSSION AND PROPOSED FUTURE WORK}

We have presented a new model for the formation of metalpoor globular clusters (MP GCs), whereby they form in the dense streams of gas predicted to feed massive galaxies at high redshift. This is reminiscent of older models for the formation of MP GCs in the halos of massive galaxies at high redshift (Fall \& Rees 1985; Cen 2001), but accounts for our new understanding of the filamentary nature of the gas in these halos. The filamentary versus spherical structure leads to higher overall gas density thus fascilitating gravitational instability. We have shown that such streams are unstable to gravitational instabilities on both small and large scales, and that such instabilities can collapse in less than a halo crossing time. At high redshift, $z \gtrsim 4$, the collapsing clouds can cool and form stars even if their metalicity is only $Z \sim 0.02 Z_{\odot}$. In the inner $\sim 0.3 R_{\mathrm{v}}$ of halos at $z \gtrsim 6$, the pressure in the streams is large enough to enable the formation of GCs. This is already true prior to the onset of gravitational instability, and will only be aided by gravitationl collapse. The mean densities and pressures in the streams at these redshifts are similar to those predicted for the ISM in a Fornax progenitor at $z \sim 3$ (Kruijssen 2015), and imply a cluster formation efficiency (CFE) of $\gtrsim 0.3-0.4$ in the streams. This value likely increases with halo mass due to increased turbulent pressure. This implies a maximal formation mass for GCs of $\sim 6 \times 10^{5} M_{\odot}$ in $\sim 10^{10} M_{\odot}$ halos at $z \sim 6$, and up to an order of magnitude larger in $10^{12} M_{\odot}$ halos. Additionally, collisions of counter-rotating streams in the inner $0.3 R_{\mathrm{v}}$ can lead to very large compression in the clouds, resulting in enough gas at high enough densities to directly form a massive GC.

Our model very naturally accounts for numerous observed properties of MP GCs and GC systems. These include the lack of dark matter in GCs, the typical masses of GCs, the correlation between the mass and metalicity of MP GCs (as opposed to MR GCs), the correlation between the mass of individual MP GCs and the mass of their host halo, the kinematics of MP GCs in the halo, the correlation of the total mass of a MP GC system with the mass of its host halo rather than the stellar mass of the central galaxy, and the correlation between the radial extent of a GC system and the virial radius of its host halo. Other predictions, such as a spread in formation times for MP GCs between $z \sim 4-7$ and a trend such that more massive, more metal rich MP GCs are younger, can be tested with future detailed observations that can increase the accuracy of age determinations for MP GCs. The key prediction of our model, that the progenitors of MP GCs should trace the streams in the halos around galaxies, out to distances of tens of kpc, will be testable with upcoming JWST observations.

Despite the many attractive features of our proposed scenario, we highlight that at the moment the evidence is merely suggestive. In \$4 we have attempted to make quantitative predictions regarding the structure and stability of cold streams and the substructure that may form within them, but these are only crude estimates. Our model demonstrates that the dense gas in cold streams is likely gravitationally unstable and can lead to the formation of stars and stellar clusters in the streams far from the central galaxy. This aspect seems supported by cosmological simulations and possibly by recent observations. However, we have not estimated the total expected SFR or CFE in the streams, nor have we shown that stream collisions in the inner halo will result in the formation of a GC. To properly address the latter, and to better understand the general process of gravitational fragmentation of streams in the halo, high resolution numerical simulations will be necessary, as detailed below. Additionally, our picture does not account at all for the MR GCs, which likely form inside galaxies (Forbes et al. 1997; Shapiro et al. 2010; Kruijssen 2015) rather than in their halos. Our main purpose in this paper was to address the general concept of stream instability at high redshift, to highlight a mechanism for the formation of MP GCs directly in the halos of massive galaxies at high redshift, and to point to supportive evidence that this scenaro is feasible and can account for many observed properties of the MP GC population.

In order to test and refine some of our assumptions and conclusions, future detailed studies of streams will be necessary. One aspect of such studies should utilise large scale cosmological simulations to obtain statistics regarding the large scale properties of both dark matter filaments and their associated gas streams, such as their mass per unit length, radial size, and spin parameter, in much the same way as similar studies have been done for dark matter halos and galaxies. By studying such global properties of filaments and how they relate to the properties of the dark matter halos they feed, the predictions of $\$ 4$ can be tested and refined. Additionally, in a follow-up paper we will examine the frequency and properties of star-forming clumps formed in the streams of the full VELA simulation suite.

The second aspect of future studies of cold streams requires detailed studies of the evolution and stability of these streams under various conditions. Since the resolution in streams in current cosmological simulations is limited (Nelson et al. 2016), such studies require idealized simulations of isolated streams inside individual halos. A few such studies have been presented recently (Mandelker et al. 2016; Cornuault et al. 2016; Padnos et al. 2018). They have shown that streams may be unstable to both hydrodynamical and thermal instabilities, which may lead to supersonic turbulence within the streams and to their eventual fragmentation. However, the analysis presented in Mandelker et al. (2016) and Padnos et al. (2018), did not include cooling, the study presented in Cornuault et al. (2016) included only analytical calculations with no simulations at all and neglected hydrodynamic instabilities, and none of these studies accounted for self gravity in the stream or the external gravitational potential of the dark matter halo. Additionally, it will be important to directly simulate the collision of counter-rotating streams, since this is predicted to be the main mechanism of GC formation in our model. These additional physics should be addressed in future work, which 
will enable the fundamental assumptions and predictions of the model presented here to be tested. Until that time, the model presented in this paper may serve as a qualitative guide to future studies of the stability of cold streams feeding massive galaxies at high redshift, the origin of MP GCs, and the relation between them.

\section{ACKNOWLEDGMENTS}

We thank the anonymous referee for constructive comments which have improved the quality of this manuscript. We are greatly indebted to Avishai Dekel for inspiring large parts of this work, and for his continuous support. We thank Avishai Dekel and Joel Primack for the use of the VELA simulations. We gratefully acknowledge Avishai Dekel, Diederik Kruijssen, and Daisuke Nagai for their careful reading of and thoughtful comments on an earlier version of the mauscript. We thank Yuval Birnboim, Frederic Bournaud, Andi Burkert, Bruce Elmegreen, Kohei Inayoshi, Thorsten Naab, and Stefanie Walch for very helpful discussions. We thank Andrew P. Hearin for his help with the Colossus package used in creating Fig.11 NM and FB acknowledge support from the Klaus Tschira Foundation, through the HITS-Yale Program in Astrophysics (HYPA). FB is supported by the US National Science Foundation through grant AST-1516962. JB acknowleges support from NSF grant AST-1616598. DC has been funded by the ERC Advanced Grant, STARLIGHT: Formation of the First Stars (project number 339177). The VELA simulations were performed at the National Energy Research Scientific Computing centre (NERSC), Lawrence Berkeley National Laboratory, and at NASA Advanced Supercomputing (NAS) at NASA Ames Research Center (PI: Joel Primack).

\section{REFERENCES}

Adamo, A., Kruijssen, J. M. D., Bastian, N., Silva-Villa, E., \& Ryon, J. 2015, MNRAS, 452, 246

Agnello, A., Evans, N. W., Romanowsky, A. J., \& Brodie, J. P. 2014, MNRAS, 442, 3299

Arrigoni Battaia, F., Hennawi, J. F., Prochaska, J. X., \& Cantalupo, S. 2015, ApJ, 809, 163

Ashman, K. M. \& Zepf, S. E. 1992, ApJ, 384, 50

Bailin, J. \& Harris, W. E. 2009, ApJ, 695, 1082

Bastian, N. 2008, MNRAS, 390, 759

Baumgardt, H. 1998, A\&A, 330, 480

Behroozi, P. S., Wechsler, R. H., \& Conroy, C. 2013, ApJ, 770, 57

Binney, J. \& Tremaine, S. 2008, Galactic Dynamics: Second Edition (Princeton University Press)

Birnboim, Y. \& Dekel, A. 2011, MNRAS, 415, 2566

Birnboim, Y., Padnos, D., \& Zinger, E. 2016, ApJL, 832, L4

Bond, J. R., Kofman, L., \& Pogosyan, D. 1996, Nature, 380, 603

Borisova, E., Cantalupo, S., Lilly, S. J., Marino, R. A., Gallego, S. G.,

Bacon, R., Blaizot, J., Bouché, N., Brinchmann, J., Carollo, C. M., Caruana, J., Finley, H., Herenz, E. C., Richard, J., Schaye, J., Straka, L. A., Turner, M. L., Urrutia, T., Verhamme, A., \& Wisotzki, L. 2016, ApJ, 831, 39

Bouché, N., Finley, H., Schroetter, I., Murphy, M. T., Richter, P., Bacon, R., Contini, T., Richard, J., Wendt, M., Kamann, S., Epinat, B., Cantalupo, S. Straka, L. A., Schaye, J., Martin, C. L., Péroux, C., Wisotzki, L., Soto, K., Lilly, S., Carollo, C. M., Brinchmann, J., \& Kollatschny, W. 2016, ApJ, 820,121

Bouché, N., Murphy, M. T., Kacprzak, G. G., Péroux, C., Contini, T., Martin, C. L., \& Dessauges-Zavadsky, M. 2013, Science, 341, 50

Boylan-Kolchin, M. 2017, ArXiv e-prints

Brodie, J. P. \& Huchra, J. P. 1991, ApJ, 379, 157

Brodie, J. P. \& Strader, J. 2006, ARA\&A, 44, 193

Brodie, J. P., Usher, C., Conroy, C., Strader, J., Arnold, J. A., Forbes, D. A., \& Romanowsky, A. J. 2012, ApJL, 759, L33

Bullock, J. S., Dekel, A., Kolatt, T. S., Kravtsov, A. V., Klypin, A. A., Porciani, C., \& Primack, J. R. 2001, ApJ, 555, 240

Cantalupo, S., Arrigoni-Battaia, F., Prochaska, J. X., Hennawi, J. F., \& Madau, P. 2014, Nature, 506, 63

Cen, R. 2001, ApJ, 560, 592

Ceverino, D., Dekel, A., \& Bournaud, F. 2010, MNRAS, 404, 2151

Ceverino, D. \& Klypin, A. 2009, ApJ, 695, 292
Ceverino, D., Klypin, A., Klimek, E. S., Trujillo-Gomez, S., Churchill, C. W., Primack, J., \& Dekel, A. 2014, MNRAS, 442, 1545

Ceverino, D., Sánchez Almeida, J., Muñoz Tuñón, C., Dekel, A., Elmegreen, B. G., Elmegreen, D. M., \& Primack, J. 2016, MNRAS, 457, 2605 Chandar, R., Fall, S. M., \& Whitmore, B. C. 2015, ApJ, 810, 1

Chandar, R., Fall, S. M., Whitmore, B. C., \& Mulia, A. J. 2017, ApJ, 849, 128

Chandrasekhar, S. \& Fermi, E. 1953, ApJ, 118, 116

Clarke, S. D., Whitworth, A. P., Duarte-Cabral, A., \& Hubber, D. A. 2017, MNRAS, 468, 2489

Clarke, S. D., Whitworth, A. P., \& Hubber, D. A. 2016, MNRAS, 458, 319

Codis, S., Pichon, C., Devriendt, J., Slyz, A., Pogosyan, D., Dubois, Y., \& Sousbie, T. 2012, MNRAS, 427, 3320

Codis, S., Pichon, C., \& Pogosyan, D. 2015, MNRAS, 452, 3369

Conroy, C., Loeb, A., \& Spergel, D. N. 2011, ApJ, 741, 72

Cornuault, N., Lehnert, M., Boulanger, F., \& Guillard, P. 2016, ArXiv e-prints

Danovich, M., Dekel, A., Hahn, O., Ceverino, D., \& Primack, J. 2015, MNRAS, 449, 2087

Danovich, M., Dekel, A., Hahn, O., \& Teyssier, R. 2012, MNRAS, 422, 1732

Dekel, A. \& Birnboim, Y. 2006, MNRAS, 368, 2

-. 2008, MNRAS, 383, 119

Dekel, A., Birnboim, Y., Engel, G., Freundlich, J., Goerdt, T., Mumcuoglu, M., Neistein, E., Pichon, C., Teyssier, R., \& Zinger, E. 2009a, Nature, 457,451

Dekel, A. \& Mandelker, N. 2014, MNRAS, 444, 2071

Dekel, A., Sari, R., \& Ceverino, D. 2009b, ApJ, 703, 785

Dekel, A., Zolotov, A., Tweed, D., Cacciato, M., Ceverino, D., \& Primack, J. R. 2013, MNRAS, 435, 999

Diemer, B. 2015, Colossus: COsmology, haLO, and large-Scale StrUcture toolS, Astrophysics Source Code Library

Diemer, B. \& Kravtsov, A. V. 2015, ApJ, 799, 108

Dijkstra, M. \& Loeb, A. 2009, MNRAS, 400, 1109

Durrell, P. R., Côté, P., Peng, E. W., Blakeslee, J. P., Ferrarese, L., Mihos, J. C., Puzia, T. H., Lançon, A., Liu, C., Zhang, H., Cuillandre, J.-C., McConnachie, A., Jordán, A., Accetta, K., Boissier, S., Boselli, A.,

Courteau, S., Duc, P.-A., Emsellem, E., Gwyn, S., Mei, S., \& Taylor, J. E. 2014, ApJ, 794, 103

El-Badry, K., Quataert, E., Weisz, D. R., Choksi, N., \& Boylan-Kolchin, M. 2018, ArXiv e-prints

Elmegreen, B. G. 2010, ApJL, 712, L184

Elmegreen, B. G., Bournaud, F., \& Elmegreen, D. M. 2008, ApJ, 688, 67

Elmegreen, B. G. \& Efremov, Y. N. 1997, ApJ, 480, 235

Elmegreen, B. G., Malhotra, S., \& Rhoads, J. 2012, ApJ, 757, 9

Emonts, B. H. C., Lehnert, M. D., Villar-Martín, M., Norris, R. P., Ekers, R. D., van Moorsel, G. A., Dannerbauer, H., Pentericci, L., Miley, G. K., Allison, J. R., Sadler, E. M., Guillard, P., Carilli, C. L., Mao, M. Y., Röttgering, H. J. A., De Breuck, C., Seymour, N., Gullberg, B., Ceverino, D., Jagannathan, P., Vernet, J., \& Indermuehle, B. T. 2016, Science, 354, 1128

Fakhouri, O., Ma, C.-P., \& Boylan-Kolchin, M. 2010, MNRAS, 406, 2267

Fall, S. M. \& Chandar, R. 2012, ApJ, 752, 96

Fall, S. M. \& Rees, M. J. 1985, ApJ, 298, 18

Fall, S. M. \& Zhang, Q. 2001, ApJ, 561, 751

Farina, E. P., Venemans, B. P., Decarli, R., Hennawi, J. F., Walter, F. Bañados, E., Mazzucchelli, C., Cantalupo, S., Arrigoni-Battaia, F., \& McGreer, I. D. 2017, ArXiv e-prints

Faucher-Giguère, C.-A., Feldmann, R., Quataert, E., Kereš, D., Hopkins, P. F., \& Murray, N. 2016, MNRAS, 461, L32

Faucher-Giguère, C.-A., Kereš, D., Dijkstra, M., Hernquist, L., \& Zaldarriaga, M. 2010, ApJ, 725, 633

Faucher-Giguère, C.-A., Kereš, D., \& Ma, C.-P. 2011, MNRAS, 417, 2982

Federrath, C., Roman-Duval, J., Klessen, R. S., Schmidt, W., \& Mac Low, M.-M. 2010, A\&A, 512, A81

Fillmore, J. A. \& Goldreich, P. 1984, ApJ, 281, 1

Forbes, D. A. 2017, ArXiv e-prints

Forbes, D. A., Brodie, J. P., \& Grillmair, C. J. 1997, AJ, 113, 1652

Forbes, D. A., Pastorello, N., Romanowsky, A. J., Usher, C., Brodie, J. P., \& Strader, J. 2015, MNRAS, 452, 1045

Forbes, D. A., Ponman, T., \& O'Sullivan, E. 2012, MNRAS, 425, 66

Forte, J. C., Vega, E. I., \& Faifer, F. 2009, MNRAS, 397, 1003

Freundlich, J., Jog, C. J., \& Combes, F. 2014, A\&A, 564, A7

Fu, H., Hennawi, J. F., Prochaska, J. X., Mutel, R., Casey, C., Cooray, A., Kereš, D., Zhang, Z.-Y., Clements, D., Isbell, J., Lang, C., McGinnis, D., Michałowski, M. J., Mooley, K., Perley, D., Stockton, A., \& Thompson, D. 2016, ApJ, 832, 52

Fumagalli, M., Prochaska, J. X., Kasen, D., Dekel, A., Ceverino, D., \& Primack, J. R. 2011, MNRAS, 418, 1796

Genel, S., Dekel, A., \& Cacciato, M. 2012, MNRAS, 3466

Gieles, M. \& Renaud, F. 2016, MNRAS, 463, L103

Ginolfi, M., Maiolino, R., Nagao, T., Carniani, S., Belfiore, F., Cresci, G., Hatsukade, B., Mannucci, F., Marconi, A., Pallottini, A., Schneider, R., \& Santini, P. 2017, MNRAS, 468, 3468

Goddard, Q. E., Bastian, N., \& Kennicutt, R. C. 2010, MNRAS, 405, 857

Goerdt, T. \& Ceverino, D. 2015, MNRAS, 450, 3359

Goerdt, T., Ceverino, D., Dekel, A., \& Teyssier, R. 2015, MNRAS, 454, 637 
Goerdt, T., Dekel, A., Sternberg, A., Ceverino, D., Teyssier, R., \& Primack,

J. R. 2010, MNRAS, 407, 613

Goerdt, T., Dekel, A., Sternberg, A., Gnat, O., \& Ceverino, D. 2012, MNRAS, 424, 2292

Harford, A. G. \& Hamilton, A. J. S. 2011, MNRAS, 416, 2678

Harris, W. E., Blakeslee, J. P., \& Harris, G. L. H. 2017, ApJ, 836, 67

Harris, W. E., Harris, G. L., \& Hudson, M. J. 2015, ApJ, 806, 36

Harris, W. E., Harris, G. L. H., \& Alessi, M. 2013, ApJ, 772, 82

Harris, W. E., Whitmore, B. C., Karakla, D., Okoń, W., Baum, W. A., Hanes, D. A., \& Kavelaars, J. J. 2006, ApJ, 636, 90

Heigl, S., Burkert, A., \& Gritschneder, M. 2017, ArXiv e-prints

Heitsch, F. 2013, ApJ, 769, 115

Hopkins, P. F. 2013, MNRAS, 430, 1653

Hudson, M. J., Harris, G. L., \& Harris, W. E. 2014, ApJL, 787, L5

Hudson, M. J. \& Robison, B. 2017, ArXiv e-prints

Inutsuka, S.-I. \& Miyama, S. M. 1992, ApJ, 388, 392

Jog, C. J. 2013, MNRAS, 434, L56

Johnson, L. C., Seth, A. C., Dalcanton, J. J., Beerman, L. C., Fouesneau, M., Lewis, A. R., Weisz, D. R., Williams, B. F., Bell, E. F., Dolphin, A. E., Larsen, S. S., Sandstrom, K. \& Skillman, E. D. 2016, ApJ, 827, 33

Kereš, D. \& Hernquist, L. 2009, ApJL, 700, L1

Kereš, D., Katz, N., Weinberg, D. H., \& Davé, R. 2005, MNRAS, 363, 2

Kim, J.-h., Ma, X., Grudić, M. Y., Hopkins, P. F., Hayward, C. C., Wetzel, A., Faucher-Giguère, C.-A., Kereš, D., Garrison-Kimmel, S., \& Murray, N. 2017, ArXiv e-prints

Kimm, T., Cen, R., Rosdahl, J., \& Yi, S. K. 2016, ApJ, 823, 52

Kimm, T., Devriendt, J., Slyz, A., Pichon, C., Kassin, S. A., \& Dubois, Y. 2011, ArXiv e-prints

Klessen, R. S. \& Hennebelle, P. 2010, A\&A, 520, A17

Konstandin, L., Girichidis, P., Federrath, C., \& Klessen, R. S. 2012, ApJ, 761,149

Kravtsov, A. V. 2003, ApJL, 590, L1

Kravtsov, A. V. \& Gnedin, O. Y. 2005, ApJ, 623, 650

Kravtsov, A. V., Klypin, A. A., \& Khokhlov, A. M. 1997, ApJS, 111, 73

Kruijssen, J. M. D. 2012, MNRAS, 426, 3008

-. 2014, Classical and Quantum Gravity, 31, 244006

-. 2015, MNRAS, 454, 1658

Kruijssen, J. M. D., Maschberger, T., Moeckel, N., Clarke, C. J., Bastian, N., \& Bonnell, I. A. 2012, MNRAS, 419, 841

Krumholz, M. R. 2012, ApJ, 759, 9

Laigle, C., Pichon, C., Codis, S., Dubois, Y., Le Borgne, D., Pogosyan, D., Devriendt, J., Peirani, S., Prunet, S., Rouberol, S., Slyz, A., \& Sousbie, T. 2015, MNRAS, 446, 2744

Larsen, S. S., Forbes, D. A., \& Brodie, J. P. 2001, MNRAS, 327, 1116

Li, H. \& Gnedin, O. Y. 2014, ApJ, 796, 10

Li, H., Gnedin, O. Y., Gnedin, N. Y., Meng, X., Semenov, V. A., \& Kravtsov, A. V. 2017, ApJ, 834, 69

Longmore, S. N., Kruijssen, J. M. D., Bastian, N., Bally, J., Rathborne, J., Testi, L., Stolte, A., Dale, J., Bressert, E., \& Alves, J. 2014, Protostars and Planets VI, 291

Mandelker, N., Dekel, A., Ceverino, D., DeGraf, C., Guo, Y., \& Primack, J. 2017, MNRAS, 464, 635

Mandelker, N., Padnos, D., Dekel, A., Birnboim, Y., Burkert, A., Krumholz, M. R., \& Steinberg, E. 2016, MNRAS, 463, 3921

Marín-Franch, A., Aparicio, A., Piotto, G., Rosenberg, A., Chaboyer, B. Sarajedini, A., Siegel, M., Anderson, J., Bedin, L. R., Dotter, A., Hempel, M., King, I., Majewski, S., Milone, A. P., Paust, N., \& Reid, I. N. 2009, ApJ, 694, 1498

Martin, D. C., Chang, D., Matuszewski, M., Morrissey, P., Rahman, S., Moore, A., \& Steidel, C. C. 2014a, ApJ, 786, 106

Martin, D. C., Chang, D., Matuszewski, M., Morrissey, P., Rahman, S., Moore, A., Steidel, C. C., \& Matsuda, Y. 2014b, ApJ, 786, 107

Matsuda, Y., Yamada, T., Hayashino, T., Yamauchi, R., \& Nakamura, Y. 2006, ApJL, 640, L123

Matsuda, Y., Yamada, T., Hayashino, T., Yamauchi, R., Nakamura, Y., Morimoto, N., Ouchi, M., Ono, Y., Kousai, K., Nakamura, E., Horie, M., Fujii, T., Umemura, M., \& Mori, M. 2011, MNRAS, 410, L13

McLaughlin, D. E. \& Fall, S. M. 2008, ApJ, 679, 1272

Mo, H. J., Yang, X., van den Bosch, F. C., \& Katz, N. 2005, MNRAS, 363, 1155

Moore, B. 1996, ApJL, 461, L13

Moore, B., Diemand, J., Madau, P., Zemp, M., \& Stadel, J. 2006, MNRAS, 368,563

Mulia, A. J., Chandar, R., \& Whitmore, B. C. 2016, ApJ, 826, 32

Muratov, A. L. \& Gnedin, O. Y. 2010, ApJ, 718, 1266

Nelson, D., Genel, S., Pillepich, A., Vogelsberger, M., Springel, V., \& Hernquist, L. 2016, MNRAS, 460, 2881

Ocvirk, P., Pichon, C., \& Teyssier, R. 2008, MNRAS, 390, 1326

Ostriker, J. 1964, ApJ, 140, 1056

Padnos, D., Mandelker, N., Birnboim, Y., Dekel, A., Krumholz, M. R., \& Steinberg, E. 2018, MNRAS, 477, 3293

Pallottini, A., Ferrara, A., Gallerani, S., Vallini, L., Maiolino, R., \& Salvadori, S. 2017, MNRAS, 465, 2540
Peebles, P. J. E. 1984, ApJ, 277, 470

Peng, E. W., Jordán, A., Côté, P., Blakeslee, J. P., Ferrarese, L., Mei, S., West, M. J., Merritt, D., Milosavljević, M., \& Tonry, J. L. 2006, ApJ, 639, 95

Pichon, C., Pogosyan, D., Kimm, T., Slyz, A., Devriendt, J., \& Dubois, Y. 2011, MNRAS, 418, 2493

Pota, V., Brodie, J. P., Bridges, T., Strader, J., Romanowsky, A. J., Villaume, A., Jennings, Z., Faifer, F. R., Pastorello, N., Forbes, D. A., Campbell, A., Usher, C., Foster, C., Spitler, L. R., Caldwell, N., Forte, J. C., Norris, M. A., Zepf, S. E., Beasley, M. A., Gebhardt, K., Hanes, D. A., Sharples, R. M., \& Arnold, J. A. 2015a, MNRAS, 450, 1962

Pota, V., Forbes, D. A., Romanowsky, A. J., Brodie, J. P., Spitler, L. R., Strader, J., Foster, C., Arnold, J. A., Benson, A., Blom, C., Hargis, J. R., Rhode, K. L., \& Usher, C. 2013, MNRAS, 428, 389

Pota, V., Romanowsky, A. J., Brodie, J. P., Peñarrubia, J., Forbes, D. A., Napolitano, N. R., Foster, C., Walker, M. G., Strader, J., \& Roediger, J. C. 2015b, MNRAS, 450, 3345

Press, W. H. \& Schechter, P. 1974, ApJ, 187, 425

Prieto, J. L. \& Gnedin, O. Y. 2008, ApJ, 689, 919

Rauch, M., Becker, G. D., Haehnelt, M. G., Carswell, R. F., \& Gauthier, J.-R. 2013, MNRAS, 431, L68

Reina-Campos, M. \& Kruijssen, J. M. D. 2017, MNRAS, 469, 1282

Renaud, F., Agertz, O., \& Gieles, M. 2017, MNRAS, 465, 3622

Renzini, A. 2017, ArXiv e-prints

Rosenblatt, E. I., Faber, S. M., \& Blumenthal, G. R. 1988, ApJ, 330, 191

Scannapieco, E., Weisheit, J., \& Harlow, F. 2004, ApJ, 615, 29

Shapiro, K. L., Genzel, R., \& Förster Schreiber, N. M. 2010, MNRAS, 403, L36

Sokolowska, A., Babul, A., Mayer, L., Shen, S., \& Madau, P. 2017, ArXiv e-prints

Spitler, L. R. \& Forbes, D. A. 2009, MNRAS, 392, L1

Spitler, L. R., Larsen, S. S., Strader, J., Brodie, J. P., Forbes, D. A., \& Beasley, M. A. 2006, AJ, 132, 1593

Springel, V., White, S. D. M., Jenkins, A., Frenk, C. S., Yoshida, N., Gao, L., Navarro, J., Thacker, R., Croton, D., Helly, J., Peacock, J. A., Cole, S., Thomas, P., Couchman, H., Evrard, A., Colberg, J., \& Pearce, F. 2005, Nature, 435, 629

Steidel, C. C., Adelberger, K. L., Shapley, A. E., Pettini, M., Dickinson, M., \& Giavalisco, M. 2000, ApJ, 532, 170

Stewart, K. R., Brooks, A. M., Bullock, J. S., Maller, A. H., Diemand, J., Wadsley, J., \& Moustakas, L. A. 2013, ApJ, 769, 74

Stewart, K. R., Kaufmann, T., Bullock, J. S., Barton, E. J., Maller, A. H., Diemand, J., \& Wadsley, J. 2011, ApJ, 738, 39

Strader, J., Brodie, J. P., Spitler, L., \& Beasley, M. A. 2006, AJ, 132, 2333

Strader, J., Romanowsky, A. J., Brodie, J. P., Spitler, L. R., Beasley, M. A., Arnold, J. A., Tamura, N., Sharples, R. M., \& Arimoto, N. 2011, ApJS, 197,33

Strader, J. \& Smith, G. H. 2008, AJ, 136, 1828

Tillson, H., Devriendt, J., Slyz, A., Miller, L., \& Pichon, C. 2015, MNRAS, 449,4363

Tormen, G., Diaferio, A., \& Syer, D. 1998, MNRAS, 299, 728

van de Voort, F. \& Schaye, J. 2012, MNRAS, 423, 2991

van de Voort, F., Schaye, J., Altay, G., \& Theuns, T. 2012, MNRAS, 421, 2809

van de Voort, F., Schaye, J., Booth, C. M., Haas, M. R., \& Dalla Vecchia, C. 2011, MNRAS, 414, 2458

van den Bosch, F. C., Jiang, F., Hearin, A., Campbell, D., Watson, D., \& Padmanabhan, N. 2014, MNRAS, 445, 1713

van Dokkum, P., Abraham, R., Romanowsky, A. J., Brodie, J., Conroy, C., Danieli, S., Lokhorst, D., Merritt, A., Mowla, L., \& Zhang, J. 2017, ArXiv e-prints

VandenBerg, D. A., Brogaard, K., Leaman, R., \& Casagrande, L. 2013, ApJ, 775,134

Vernet, J., Lehnert, M. D., De Breuck, C., Villar-Martín, M., Wylezalek, D., Falkendal, T., Drouart, G., Kolwa, S., Humphrey, A., Venemans, B. P., \& Boulanger, F. 2017, A\&A, 602, L6

Walker, D. L., Longmore, S. N., Bastian, N., Kruijssen, J. M. D., Rathborne, J. M., Jackson, J. M., Foster, J. B., \& Contreras, Y. 2015, MNRAS, 449, 715

Webb, J. J. \& Leigh, N. W. C. 2015, MNRAS, 453, 3278

Wehner, E. M. H., Harris, W. E., Whitmore, B. C., Rothberg, B., \& Woodley, K. A. 2008, ApJ, 681, 1233

Yang, X., Mo, H. J., \& van den Bosch, F. C. 2003, MNRAS, 339, 1057

Zel'dovich, Y. B. 1970, A\&A, 5, 84

Zhao, D. H., Jing, Y. P., Mo, H. J., \& Börner, G. 2009, ApJ, 707, 354

Zolotov, A., Dekel, A., Mandelker, N., Tweed, D., Inoue, S., DeGraf, C. Ceverino, D., Primack, J. R., Barro, G., \& Faber, S. M. 2015, MNRAS, 450,2327 


\section{APPENDIX}

\section{A. STREAM RADIUS AND DENSITY IN THE CASE OF NO ROTATION SUPPORT}

In $\$ 4.1$ we derived expressions for the typical radius and density of cold streams assuming a constant ratio of stream radius to the radius of the host dark-matter filament, and that the streams were fully rotationally supported. Here we examine the stream radius and density in the case of no rotation, assuming purely radial accretion onto the streams from the sheets within which they are embedded, and show that the results are inconsistent with both cosmological simulations and observations.

By combining eqs. (3) and (9) for the stream line-mass, we obtain one equation relating the stream radius and density,

$$
r_{\mathrm{s}}^{2} \rho_{\mathrm{s}} \simeq 6.1 \times 10^{6} M_{\odot} \mathrm{kpc}^{-1} M_{10}^{0.77}(1+z)_{7}^{2} f_{\mathrm{s}, 3} \mathcal{M}_{\mathrm{v}}{ }^{-1} .
$$

A second equation can be obtained by considering the case of radial accretion onto the stream from a background with density $\rho_{\text {acc }}$ flowing at velocity $V_{\text {acc }}$ towards the stream axis. The accretion rate onto the stream in this case can be written as

$$
\dot{M}_{\mathrm{L}, \mathrm{s}}=2 \pi r_{\mathrm{s}} V_{\text {acc }} \rho_{\text {acc }} \text {. }
$$

The accretion rate onto the stream can be obtained by taking the derivative of eq. (9) with respect to time, and inserting eq. (4) as well as $(1+z)_{7} \simeq(t / 0.95 \mathrm{Gyr})^{-2 / 3}$, and is given by eq. (31). The accretion velocity is given by the gravitational potential of the dark-matter filament and the gas stream, and is given by eq. 30 . To within a factor of $\lesssim 2$, this is the virial velocity of the dark-matter filament.

What remains is to relate the density in the stream, $\rho_{\mathrm{s}}$, to that of the accreted material, $\rho_{\mathrm{acc}}$. Since the cooling time in the sheets is short, the accreted gas is isothermal (Mo et al. 2005) and we expect a strong accretion shock at the stream boundary where the density increases by a factor $\mathcal{M}_{\mathrm{acc}}^{2}$, where

$$
\mathcal{M}_{\mathrm{acc}}=V_{\mathrm{acc}} / c_{\mathrm{s}} \simeq 7.8 M_{10}^{0.38}(1+z)_{7} f_{\mathrm{s}, 3}^{0.5} \mathcal{M}_{\mathrm{v}}{ }^{-0.5} T_{4}^{-0.5} .
$$

is the Mach number of the accreted material. We thus have $\rho_{\mathrm{s}} \simeq \mathcal{M}_{\mathrm{acc}}^{2} \rho_{\text {acc }}$. Putting all this together, we obtain a second equation relating the stream radius and density:

$$
r_{\mathrm{s}} \rho_{\mathrm{s}} \simeq 7.9 \times 10^{6} M_{\odot} \mathrm{kpc}^{-2} M_{10}^{1.15}(1+z)_{7}^{4.5} f_{\mathrm{s}, 3}^{1.5} \mathcal{M}_{\mathrm{v}}{ }^{-1.5} T_{4}^{-1} .
$$

Combining eqs. (A1) and A4 we obtain

$$
r_{\mathrm{s}} \simeq 0.8 \mathrm{kpc} M_{10}^{-0.38}(1+z)_{7}^{-2.5} f_{\mathrm{s}, 3}^{-0.5} \mathcal{M}_{\mathrm{v}}^{2.5} T_{4}
$$

and

$$
n_{\mathrm{H}, \mathrm{s}} \simeq 0.3 \mathrm{~cm}^{-3} M_{10}^{1.53}(1+z)_{7}^{7} f_{\mathrm{s}, 3}^{2} \mathcal{M}_{\mathrm{v}}{ }^{-4} T_{4}^{-2} .
$$

Note that these are the values at $R_{\mathrm{v}}$, before the stream enters the halo. Within the halo, the radius scales as $x_{\mathrm{v}}=R / R_{\mathrm{v}}$, while the density scales as $x_{\mathrm{v}}^{-2}$.

Somewhat amazingly, the above estimate assuming purely radial accretion and the estimate from $\$ 4.1$ assuming total rotational support both agree for a $10^{10} M_{\odot}$ halo at $z=6$. However, when scaled to a $10^{12} M_{\odot}$ halo at $z=3$, the above estimate yields densities of $n_{\mathrm{H} . \mathrm{s}} \sim 6.85 \mathrm{~cm}^{-3}$, several orders of magnitude larger than the densities seen in cosmological simulations (e.g. Goerdt et al. 2010; Faucher-Giguère et al. 2010; van de Voort \& Schave 2012), and inferred from observations of giant Ly $\alpha$ nebulae around massive galaxies at $z \sim 3$ (e.g Cantalupo et al. 2014; Arrigoni Battaia et al. 2015; Borisova et al. 2016; Ginolfi et al. 2017). Furthermore, for the same halo mass and redshift eq. A5] results in a ratio of stream radius to halo virial radius of $r_{\mathrm{s}} / R_{\mathrm{v}} \sim 7 \times 10^{-3}$, significantly smaller than what is seen in simulations. Indeed, such small streams are predicted to rapidly disrupt in the halo due to KelvinHelmholz Instabilities (Mandelker et al. 2016; Padnos et al., in preparation).

We conclude that the streams are not fed by purely radial accretion from their surroundings, and that the accreted material must have some rotational support. This is consistent with the large vorticities present in the stream gas (Codis et al. 2012, 2015; Laigle et al. 2015), and justifies our derivation of the stream radius and density assuming rotational support in \$4.1]

\section{B. EFFECT OF A TIDAL FIELD ON THE FRAGMENTATION SCALE}

In $\$ 4.3$ we derived the characteristic fragmentation scale in the streams using the Jeans stability criterion. However, in the presence of a tidal field these scales may be modified. The modified Jeans length can be approximated as (Jog 2013)

$$
\lambda_{\mathrm{j}}^{\prime}=\frac{L_{\mathrm{J}}}{\left[1-T_{0} /\left(4 \pi G \rho_{0}\right)\right]^{1 / 2}},
$$

where $L_{\mathrm{J}}$ is the Jeans length in the absence of the tidal field (eq. (36) $), \rho_{0}$ is the unperturbed background density, and $T_{0}=\partial^{2} \Phi / \partial r^{2}$ is the second derivative of the gravitational potential, estimated at the center of the region. If $T_{0}>0$ then the tidal field is extensive and the fragmentation scale length is larger than in the case with no tidal field. If $T_{0}<0$ the tidal field is compressive and the fragmentation scale decreases. There are two sources of tidal forces on a cloud in the center of the streams: the dark matter halo and the dark matter filament. We estimate the ratio $T_{0} /\left(4 \pi G \rho_{\mathrm{s}}\right)$ for each of these. 
For the halo, we assume it is an isothermal sphere with $M(r) \propto r$, resulting in $T_{0, \text { halo }}=\left(V_{\mathrm{v}} / R_{\mathrm{v}}\right)^{2} x_{\mathrm{v}}^{-2}$. Together with eqs. (7), (8) and (24) this yields

$$
\frac{T_{0, \text { halo }}}{4 \pi G \rho_{\mathrm{s}}} \simeq 0.08 \mathrm{E} 1^{2} .
$$

This is independent of halo mass or redshift. For $Ł 1=1$, this results in a $\sim 4 \%$ increase in the Jeans length relative to eq. (37), and a corresponding $\sim 12 \%$ increase in the Jeans mass relative to eq. 38). For $€ 1=0.5$, as was often assumed in our analysis, the difference is even smaller. This is negligible relative to the other uncertainties in our model.

For the dark matter filament, we assume that it is an isothermal filament with a line-mass profile given by eq. (19). This results in $T_{0, \mathrm{Fil}}=-\pi G \rho_{\mathrm{dm}, \mathrm{c}}$, where $\rho_{\mathrm{dm}, \mathrm{c}}$ is the central density of the dark matter filament. This is related to the total line-mass by $\rho_{\mathrm{dm}, \mathrm{c}}=M_{\mathrm{L}, \mathrm{Fil}} /\left(\pi r_{\mathrm{h}}^{2}\right)$, with $r_{\mathrm{h}}=\beta^{-1} r_{\mathrm{v}, \mathrm{Fil}}$ the half-mass radius of the filament. Note that since $T_{0, \mathrm{Fil}}<0$, the tidal field is compressive. Putting all this together yields

$$
\frac{\left|T_{0, \mathrm{Fil}}\right|}{4 \pi G \rho_{\mathrm{s}}} \simeq \frac{0.014 \mathrm{E} 1^{2}}{\beta^{2}} .
$$

For any $\beta>1$, the effect on the fragmentation scale is completely negligible. 